TRANSACTIONS OF THE

AMERICAN MATHEMATICAL SOCIETY

Volume 363, Number 8, August 2011, Pages 4171-4201

S 0002-9947(2011)05158-5

Article electronically published on March 15, 2011

\title{
SLOW ESCAPING POINTS OF MEROMORPHIC FUNCTIONS
}

\author{
P. J. RIPPON AND G. M. STALLARD
}

\begin{abstract}
We show that for any transcendental meromorphic function $f$ there is a point $z$ in the Julia set of $f$ such that the iterates $f^{n}(z)$ escape, that is, tend to $\infty$, arbitrarily slowly. The proof uses new covering results for analytic functions. We also introduce several slow escaping sets, in each of which $f^{n}(z)$ tends to $\infty$ at a bounded rate, and establish the connections between these sets and the Julia set of $f$. To do this, we show that the iterates of $f$ satisfy a strong distortion estimate in all types of escaping Fatou components except one, which we call a quasi-nested wandering domain. We give examples to show how varied the structures of these slow escaping sets can be.
\end{abstract}

\section{INTRODUCTION}

Let $f: \mathbb{C} \rightarrow \hat{\mathbb{C}}$ be a meromorphic function that is not rational of degree 1 , and denote by $f^{n}, n=0,1,2, \ldots$, the $n$th iterate of $f$. The Fatou set $F(f)$ is defined to be the set of points $z \in \mathbb{C}$ such that $\left(f^{n}\right)_{n \in \mathbb{N}}$ is well-defined and forms a normal family in some neighbourhood of $z$. The complement $\mathbb{C} \backslash F(f)$ is called the Julia set $J(f)$ of $f$. An introduction to the properties of these sets can be found in [7].

This paper concerns the escaping set of $f$, defined as follows:

$$
I(f)=\left\{z: f^{n}(z) \text { is defined for } n \in \mathbb{N}, f^{n}(z) \rightarrow \infty \text { as } n \rightarrow \infty\right\} .
$$

If $f$ is a polynomial, then $I(f)$ is a neighbourhood of $\infty$ in which iterates tend to $\infty$ at a uniform rate, so $I(f) \subset F(f)$ and $J(f)=\partial I(f)$; see [6]. For a transcendental entire function $f$, the escaping set was first studied by Eremenko [15] who proved that

$$
I(f) \cap J(f) \neq \emptyset,
$$

unlike for a polynomial, that

$$
J(f)=\partial I(f),
$$

as for a polynomial, and finally that

$$
\text { all components of } \overline{I(f)} \text { are unbounded. }
$$

Domínguez [14] showed that the first two of these properties are true for any transcendental meromorphic function, but the third is not. The set $I(f)$ and the dynamical behaviour of $f$ on $I(f)$ are more complicated for a transcendental meromorphic function than for a polynomial. For example, $I(f)$ can have infinitely many components or it can be connected, and simple examples show that the set $I(f)$ may or may not meet $F(f)$.

Received by the editors September 5, 2008 and, in revised form, June 23, 2009.

2010 Mathematics Subject Classification. Primary 37F10; Secondary 30D05. 
For a transcendental entire function $f$, the fast escaping set was introduced by Bergweiler and Hinkkanen in [11:

$$
A(f)=\left\{z \text { : there exists } L \in \mathbb{N} \text { such that }\left|f^{n+L}(z)\right|>M\left(R, f^{n}\right) \text {, for } n \in \mathbb{N}\right\} .
$$

Here,

$$
M(r, f)=\max _{|z|=r}|f(z)|, \quad \text { for } r>0,
$$

and $R$ is any value such that $R>\min _{z \in J(f)}|z|$. The set $A(f)$ has many properties that make it easier to work with than $I(f)$; see [11, 30] and [34. For example, all its components are unbounded, whereas for $I(f)$ this is an open question asked by Eremenko in [15]. The set $A(f)$ also meets $J(f)$, and we have $J(f)=\partial A(f)$. Note that $A(f)$ is a subset of

$$
Z(f)=\left\{z \in I(f): \frac{1}{n} \log \log \left|f^{n}(z)\right| \rightarrow \infty \text { as } n \rightarrow \infty\right\},
$$

which is the set of points that 'zip towards $\infty$ '; see [11. The set $Z(f)$ is defined for all transcendental meromorphic functions, it meets $J(f)$ and we have $J(f)=\partial Z(f)$; see [29].

It is natural to expect that $Z(f) \neq I(f)$ for every transcendental meromorphic function $f$, but this has not been established previously. Here we prove a much stronger result. We show that for all transcendental meromorphic functions $f$ there are points of $I(f)$ whose iterates tend to $\infty$ arbitrarily slowly, that is, more slowly than at any given rate.

Theorem 1. Let $f$ be a transcendental meromorphic function. Then, given any positive sequence $\left(a_{n}\right)$ such that $a_{n} \rightarrow \infty$ as $n \rightarrow \infty$, there exist

$$
\zeta \in I(f) \cap J(f) \text { and } N \in \mathbb{N}
$$

such that

$$
\left|f^{n}(\zeta)\right| \leq a_{n}, \quad \text { for } n \geq N .
$$

Our proof of Theorem 1 relies on certain new covering properties of annuli, which we state and prove in Section 2, and on the Ahlfors five islands theorem. We prove Theorem 1 for functions with finitely many poles in Section 3, and for functions with infinitely many poles in Section 4. We also indicate how the proof of Theorem 1 can be adapted to construct points such that (1.5) holds, and

$$
\liminf _{n \rightarrow \infty}\left|f^{n}(\zeta)\right|<\infty \quad \text { and } \quad \limsup _{n \rightarrow \infty}\left|f^{n}(\zeta)\right|=\infty .
$$

Rempe [25, Theorem 1.4] proved a slow escape result for the exponential family by using facts about the structure of the escaping sets of such functions, which are known to be unions of curves to infinity called dynamic rays, or hairs. Expressed in terms of functions of the form $f_{\lambda}(z)=\lambda e^{z}$, where $\lambda \neq 0$, his result states that for any positive sequence $\left(a_{n}\right)$ such that $a_{n} \rightarrow \infty$ as $n \rightarrow \infty$ and $a_{n+1}=O\left(\exp \left(a_{n}\right)\right)$ as $n \rightarrow \infty$, there is a point $\zeta \in J\left(f_{\lambda}\right)$ - in fact, an escaping endpoint of a dynamic ray - and $N \in \mathbb{N}$ such that

$$
\frac{1}{C} a_{n} \leq\left|f^{n}(\zeta)\right| \leq C a_{n}, \quad \text { for } n \geq N
$$

where $C=\exp (2+2 \pi)$.

In Section 5, we show that a two-sided slow escape result of this type holds for a wide range of meromorphic functions. 
Theorem 2. Let $f$ be a transcendental meromorphic function with a finite number of poles. Then $f$ has the property that, for all positive sequences $\left(a_{n}\right)$ such that $a_{n} \rightarrow \infty$ as $n \rightarrow \infty$ and $a_{n+1}=O\left(M\left(a_{n}, f\right)\right)$ as $n \rightarrow \infty$, there exist $\zeta \in J(f)$ and $C>1$ such that

$$
a_{n} \leq\left|f^{n}(\zeta)\right| \leq C a_{n}, \quad \text { for } n \in \mathbb{N},
$$

if and only if there are positive constants $c, d$ and $r_{0}$ such that $d>1$ and

$$
\text { for all } r \geq r_{0} \text { there exists } \rho \in(r, d r) \text { such that } m(\rho, f) \leq c \text {. }
$$

Here

$$
m(r, f)=\min _{|z|=r}|f(z)|, \quad \text { for } r>0,
$$

denotes the minimum modulus of $f$. Note that (1.7) holds whenever $f$ is bounded on some path to $\infty$.

We prove Theorem 2] in Section 5 . The proof of Theorem 2 is considerably simpler than that of Theorem 1, where extra difficulty arises from the fact that $m(r, f)$ may be large for long intervals of values of $r$. It is clear that in Theorem 2 some restriction on the sequence $\left(a_{n}\right)$ is needed, such as $a_{n+1}=O\left(M\left(a_{n}, f\right)\right)$ as $n \rightarrow \infty$. To extend Theorem 2 to more general meromorphic functions, some replacement for the restriction $a_{n+1}=O\left(M\left(a_{n}, f\right)\right)$ as $n \rightarrow \infty$ would be needed.

Now we introduce sets of points which escape to $\infty$ at various bounded rates and investigate to what extent the Eremenko properties (1.2) and (1.3) hold for these new sets. First we define the slow escaping set of a transcendental meromorphic function $f$,

$$
L(f)=\left\{z \in I(f): \limsup _{n \rightarrow \infty} \frac{1}{n} \log \left|f^{n}(z)\right|<\infty\right\},
$$

and the moderately slow escaping set of $f$,

$$
M(f)=\left\{z \in I(f): \limsup _{n \rightarrow \infty} \frac{1}{n} \log \log \left|f^{n}(z)\right|<\infty\right\} .
$$

Evidently we have $L(f) \subset M(f) \subset I(f) \backslash Z(f)$.

Next, for a positive sequence $a=\left(a_{n}\right)$ such that $a_{n} \rightarrow \infty$ as $n \rightarrow \infty$, we define

$$
I^{a}(f)=\left\{z \in I(f):\left|f^{n}(z)\right|=O\left(a_{n}\right) \text { as } n \rightarrow \infty\right\},
$$

where the constant in the $O($.$) condition depends on the point z$. If $a_{n} \rightarrow \infty$ as $n \rightarrow \infty$ and $a_{n}=O\left(C^{n}\right)$ as $n \rightarrow \infty$, for some $C>1$, then $I^{a}(f) \subset L(f)$. Note that each set $L(f), M(f)$ and $I^{a}(f)$ is non-empty and meets $J(f)$, by Theorem 1 .

To state our results about these sets we need some further notions. First we describe certain types of Fatou components. For any component $U$ of $F(f)$ we write $U_{n}$, for $n \in \mathbb{N}$, to denote the component of $F(f)$ which contains $f^{n}(U)$. Then $U$ is a wandering domain of $f$ if the sequence $U_{n}$ is not periodic or pre-periodic.

Recall from [29] that a Baker wandering domain of $f$ is a wandering domain $U$ of $f$ such that

- each $U_{n}$ is bounded,

- $U_{n}$ surrounds 0 for large $n$,

- $U_{n} \rightarrow \infty$ as $n \rightarrow \infty$.

The first example of such a wandering domain was given by Baker 1 . 
We also introduce the notion of a nested wandering domain of $f$, which is a wandering domain $U$ of $f$ such that

- each $U_{n}$ is bounded,

- $U_{n+1}$ surrounds $U_{n}$ for large $n$,

- $U_{n} \rightarrow \infty$ as $n \rightarrow \infty$.

Finally, we introduce the notion of a quasi-nested wandering domain of $f$, which is a wandering domain $U$ of $f$ such that there is a sequence $\left(n_{j}\right)$ in $\mathbb{N}$ for which

- each $U_{n}$ is bounded,

- each $U_{n_{j}}$ surrounds 0 ,

- $U_{n_{j}} \rightarrow \infty$ as $j \rightarrow \infty$.

Any nested wandering domain is a Baker wandering domain and, in turn, any Baker wandering domain is a quasi-nested wandering domain. For transcendental entire functions, any multiply connected Fatou component is a nested wandering domain, by [2, Theorem 3.1], so all three definitions are equivalent and the terms are interchangeable. An example of a transcendental meromorphic function with a quasi-nested wandering domain that is not a Baker wandering domain can be found in [14, Theorem E]; see also Example 1 of this paper. These examples could be modified to provide an example of a transcendental meromorphic function with a Baker wandering domain that is not a nested wandering domain.

Our next result includes a 'slow escape' version of Eremenko's property (1.2).

Theorem 3. Let $f$ be a transcendental meromorphic function and let $a=\left(a_{n}\right)$ be a positive sequence such that $a_{n} \rightarrow \infty$ as $n \rightarrow \infty$. Then

(a) $L(f)$ and $M(f)$ are completely invariant under $f$;

(b) $L(f), M(f)$ and $I^{a}(f)$ are each dense in $J(f)$;

(c) $J(f)=\partial L(f)=\partial M(f)$;

(d) we have

$$
\begin{aligned}
J(f) & \subset \partial I^{a}(f) \\
& \subset J(f) \cup \bigcup\{U: U \text { is a quasi-nested wandering domain }\},
\end{aligned}
$$

so if $I^{a}(f)$ meets no quasi-nested wandering domain, then $J(f)=\partial I^{a}(f)$.

Remarks. 1. If $J(f)$ is connected, then the sets

$$
\partial L(f)=\partial M(f)=J(f), \quad \overline{L(f)} \text { and } \overline{M(f)}
$$

are also connected, as are $\partial I^{a}(f)$ and $\overline{I^{a}(f)}$, provided that $I^{a}(f)$ meets no quasinested wandering domain, by Theorem 3 (c) and (d). Note that $J(f)$ is connected if and only if all Fatou components of $f$ have connected boundaries; see 23. Proposition 1].

2. In Theorem 3(d), quasi-nested wandering domains arise because they are 'exceptional' Fatou components in the following sense: all other types of Fatou components $U$ in $I(f)$ have the property that if $\Delta$ is a compact disc in $U$, then there exist $C>1$ and $n_{0} \in \mathbb{N}$ such that

$$
\left|f^{n}\left(z^{\prime}\right)\right| \leq C\left|f^{n}(z)\right|, \quad \text { for } z, z^{\prime} \in \Delta, n \geq n_{0} ;
$$

see Theorem 5 in Section 6 . 
3. For any meromorphic function $f$ and any sequence $a=\left(a_{n}\right)$ satisfying the hypotheses of Theorem 2, it is natural to consider subsets of $I(f)$ of the form

$$
\left\{z \in I(f): \text { there exists } C=C(z)>1 \text { such that } \frac{a_{n}}{C} \leq\left|f^{n}(z)\right| \leq C a_{n}\right\}
$$

and to ask if results similar to those in Theorem 3 can be obtained. Results of this type appear to depend on how fast $a_{n}$ tends to $\infty$ in relation to the speed of escape of points in $A(f)$; we hope to return to this question in a later paper.

Next we describe a family of transcendental meromorphic functions which have many dynamical properties in common with transcendental entire functions; see [12]. Let $D$ be an unbounded domain in $\mathbb{C}$ whose boundary consists of piecewise smooth curves, and suppose that $\{z:|z|>r\} \backslash D \neq \emptyset$ for all $r>0$. Let $f$ be a complexvalued function whose domain of definition contains the closure $\bar{D}$ of $D$. Then $D$ is called a direct tract of $f$ if the function $f$ is analytic in $D$ and continuous in $\bar{D}$ and if there exists $R>0$ such that $|f(z)|=R$ for $z \in \partial D$ and $|f(z)|>R$ for $z \in D$. For example, any transcendental meromorphic function with a finite number of poles has at least one direct tract. However, a transcendental meromorphic function with infinitely many poles may or may not have a direct tract.

Recently, the following results about functions with a direct tract were obtained; see [12, Theorem 5.1(a), Theorem 4.1(c) and Theorem 5.2].

Let $f$ be a transcendental meromorphic function with a direct tract:

- if $U$ is a Baker wandering domain of $f$, then $\bar{U} \subset Z(f)$;

- there is a constant $r_{0}>0$ such that if $U$ is a component of $F(f)$ which contains a Jordan curve surrounding $\left\{z:|z|=r_{0}\right\}$, then $U$ is a Baker wandering domain, and indeed a nested wandering domain.

Thus, if $f$ is a transcendental meromorphic function with a direct tract, then the notions of quasi-nested wandering domain, Baker wandering domain and nested wandering domain are equivalent. In earlier papers, we used the term Baker wandering domain to describe such a wandering domain. From now on, we will usually use the name nested wandering domain, since it is the strongest of these notions and also the most descriptive. Note that if $f$ has a direct tract, then any nested wandering domain of $f$ is in $Z(f)$ by the first bullet point above. Thus we obtain the following corollary of Theorem 3 (d).

Corollary 1. Let $f$ be a transcendental meromorphic function with a direct tract and let $a=\left(a_{n}\right)$ be a positive sequence such that $a_{n} \rightarrow \infty$ as $n \rightarrow \infty$. Then

(a) we have

$$
\begin{aligned}
J(f) & \subset \partial I^{a}(f) \\
& \subset J(f) \cup \bigcup\{U: U \text { is a nested wandering domain }\},
\end{aligned}
$$

so if $I^{a}(f)$ meets no nested wandering domain, then $J(f)=\partial I^{a}(f)$;

(b) if $\frac{1}{n} \log \log a_{n} \nrightarrow \infty$ as $n \rightarrow \infty$, then $I^{a}(f)$ meets no nested wandering domain, so $J(f)=\partial I^{a}(f)$.

We prove Theorem 3 in Section 6. We also show there that Theorem 3 (d) and Corollary 1(a) cannot be improved to state that we always have $J(f)=\partial I^{a}(f)$, and that in Corollary 1(a) the assumption about the existence of a direct tract cannot be omitted. 
Our final result includes a 'slow escape' version of Eremenko's property (1.3) for a meromorphic function with a direct tract, and it shows that a fundamental difference occurs here depending on whether or not there are nested wandering domains. First recall that if $f$ is a transcendental meromorphic function with a direct tract, then $I(f)$ has at least one unbounded component; see [12, Theorem 1.1]. However, if $f$ has no direct tract, then $\overline{I(f)}$ may have no unbounded components; for example, for $f(z)=\frac{1}{2} \tan z$ the set $\overline{I(f)}=J(f)$ is totally disconnected.

Theorem 4. Let $f$ be a transcendental meromorphic function with a direct tract.

(a) Suppose that $a=\left(a_{n}\right)$ is a positive sequence such that $a_{n} \rightarrow \infty$ as $n \rightarrow \infty$.

(i) If $f$ has no nested wandering domains, then the sets $\partial L(f), \partial M(f)$ and $\partial I^{a}(f)$ all have an unbounded component, and therefore so do $\overline{L(f)}, \overline{M(f)}$ and $\overline{I^{a}(f)}$.

(ii) If $f$ is entire and has no nested wandering domains, then all the components of $\partial L(f), \partial M(f)$ and $\partial I^{a}(f)$ are unbounded and therefore so are all the components of $\overline{L(f)}, \overline{M(f)}$ and $\overline{I^{a}(f)}$.

(b) If $f$ has a nested wandering domain, then all the components of $\overline{M(f)}$ are bounded.

We prove Theorem 4 in Section 7, and in Section 8 we give a number of examples which show how varied the structures of the sets $L(f), M(f)$ and $I^{a}(f)$ can be.

We end this section by making some observations about the possible relationships between these various subsets of the escaping set and the components of the Fatou set when $f$ is a transcendental meromorphic function.

- Any Fatou component $U$ which meets $I(f)$ must lie in $I(f)$, and such a Fatou component must be either a wandering domain or a Baker (or pre-Baker) domain; that is, $U$ eventually maps into a $p$-cycle of Fatou components in which $f^{n p}(z) \rightarrow z_{0}$ as $n \rightarrow \infty$ but $f^{p}\left(z_{0}\right)$ is not defined.

- If $f$ has a Baker domain $U \subset I(f)$, then $U \subset L(f)$, by [28, Theorem 1].

- As pointed out earlier in this section, if $f$ has a direct tract and $U$ is a nested wandering domain of $f$, then $\bar{U} \subset Z(f)$ and hence $\bar{U} \cap M(f)=\emptyset$.

- There exists a transcendental meromorphic function $f$, with no direct tract, which has a nested wandering domain $U$ such that $\bar{U} \subset L(f)$; see 31 , discussion after Theorem 3].

- There exists a transcendental entire function with a simply connected wandering domain contained in $A(f)$; see [10].

- There exists a transcendental entire function with a simply connected wandering domain (either bounded or unbounded) contained in $L(f)$; see Examples 4 and 5 in Section 8.

\section{Preliminary Results}

The construction of the slowly escaping point in Theorem 1 uses the following simple lemma.

Lemma 1. Let $E_{n}, n \geq 0$, be a sequence of compact sets in $\mathbb{C}$ and $f: \mathbb{C} \rightarrow \hat{\mathbb{C}}$ be a continuous function such that

$$
f\left(E_{n}\right) \supset E_{n+1}, \quad \text { for } n \geq 0 .
$$

Then there exists $\zeta$ such that $f^{n}(\zeta) \in E_{n}$, for $n \geq 0$. 
If $f$ is also meromorphic and $E_{n} \cap J(f) \neq \emptyset$, for $n \geq 0$, then there exists $\zeta \in J(f)$ such that $f^{n}(\zeta) \in E_{n}$, for $n \geq 0$.

Proof. Let

$$
F_{n}=\left\{z \in E_{0}: f(z) \in E_{1}, \ldots, f^{n}(z) \in E_{n}\right\} .
$$

Then, by (2.1), $F_{n}$ is a decreasing sequence of non-empty compact sets, so $F=$ $\bigcap_{n=0}^{\infty} F_{n}$ is non-empty. If $\zeta \in F$, then $f^{n}(\zeta) \in E_{n}$, for $n \geq 0$, as required.

The second statement follows by applying the first statement to the non-empty compact sets $E_{n} \cap J(f), n \geq 0$, in view of the complete invariance of $J(f)$.

In our proof of Theorem 1 for functions with a finite number of poles, we apply Lemma 1 to sets $E_{n}$ that are closed annuli. In order to do this we require two annulus covering properties. Throughout, we use the following notation, for $z \in \mathbb{C}$ and $0<r<R$ :

- $A(r, R)=\{z: r<|z|<R\}$,

- $B(z, r)=\{w:|w-z|<r\}$.

We use a result of Baker and Liverpool [4, Lemma 1].

Lemma 2. Let $f$ be analytic in the annulus $A(\alpha, \beta)$ and let $\left|z_{0}\right|=|z|=\sqrt{\alpha \beta}$. If $f$ omits the values 0 and 1 in $A(\alpha, \beta)$, then

$$
|f(z)| \leq \exp \left(\left(\log ^{+}\left|f\left(z_{0}\right)\right|+C_{0}\right)\left(\exp \left(\pi^{2} / \log \gamma\right)+1\right)\right),
$$

where $C_{0}$ is a positive absolute constant and $\gamma=\beta / \alpha$. In particular, if we also have $\gamma \geq 2$, then

$$
|f(z)| \leq\left(\left|f\left(z_{0}\right)\right|+2\right)^{L},
$$

where $L>2$ is an absolute constant.

The estimate (2.2) follows from the fact that

$$
\log ^{+} t+C \leq 2 C \log (t+2), \quad \text { for } t \geq 0, C \geq 2 .
$$

We use Lemma 2 to prove our first annulus covering property, which is related to a theorem of Bohr; see [19, page 170].

Lemma 3. Let $f$ be a transcendental meromorphic function with a finite number of poles, let $c L<1 / 4$, where $c>0$ and $L$ is the constant in Lemma 2 , and let $R_{0}=R_{0}(f)>0$ be so large that $M(r, f)$ is increasing on $\left[R_{0}, \infty\right)$ and

$$
M(r, f)>3^{4 L}, \quad \text { for } r \geq R_{0} .
$$

If $r>R_{0}$ and

$$
\text { there exists } \rho \in(2 r, 4 r) \text { such that } \log m(\rho, f) \leq c \log M(\rho, f) \text {, }
$$

then, for any $R$ and $\tilde{R}$ such that

$$
2<R \quad \text { and } \quad R^{10}<\tilde{R}<M(r, f)^{1 / 10},
$$

we have

$$
f(A(r, 8 r)) \text { covers } A\left(R, R^{5}\right) \text { or } A\left(\tilde{R}, \tilde{R}^{5}\right) \text {. }
$$


Proof. Suppose that $r>R_{0}$. By (2.4), there exists $\rho \in(2 r, 4 r)$ such that $m(\rho, f) \leq$ $M(\rho, f)^{c}$. Then $A(r, 8 r) \supset A\left(\frac{1}{2} \rho, 2 \rho\right)$.

Now suppose that $f$ omits in $A\left(\frac{1}{2} \rho, 2 \rho\right)$ two values:

$$
w_{1} \in A\left(R, R^{5}\right) \quad \text { and } \quad w_{2} \in A\left(\tilde{R}, \tilde{R}^{5}\right) .
$$

Then $w_{1} \neq w_{2}$ and

$$
g(z)=\frac{f(z)-w_{1}}{w_{2}-w_{1}}
$$

omits in $A\left(\frac{1}{2} \rho, 2 \rho\right)$ the values 0 and 1 , so we can apply (2.2) to the function $g$.

Take $z_{0}$ such that $\left|z_{0}\right|=\rho$ and $\left|f\left(z_{0}\right)\right|=m(\rho, f) \leq M(\rho, f)^{c}$. Since $\tilde{R} \geq 2 R^{5}$, we have

$$
\left|g\left(z_{0}\right)\right| \leq \frac{\left|f\left(z_{0}\right)\right|+R^{5}}{\tilde{R}-R^{5}} \leq \frac{\left|f\left(z_{0}\right)\right|+R^{5}}{R^{5}} .
$$

Therefore, for $|z|=\rho$, we have, by (2.2),

$$
\begin{aligned}
|f(z)| & \leq\left|w_{1}\right|+\left(\left|w_{2}\right|+\left|w_{1}\right|\right)|g(z)| \\
& \leq R^{5}+2 \tilde{R}^{5}\left(\left|g\left(z_{0}\right)\right|+2\right)^{L} \\
& \leq R^{5}+2 \tilde{R}^{5}\left(\frac{\left|f\left(z_{0}\right)\right|+3 R^{5}}{R^{5}}\right)^{L} \\
& \leq R^{5}+2 \tilde{R}^{5}\left(\frac{M(\rho, f)^{c}+3 R^{5}}{R^{5}}\right)^{L} .
\end{aligned}
$$

Now

$$
R<\tilde{R}<M(\rho, f)^{1 / 10}, \quad 0<c L<1 / 4 \quad \text { and } \quad 1+2^{L+1}<3^{L}<M(\rho, f)^{1 / 4},
$$

by (2.3) and the fact that $L>2$. We deduce that, for $|z|=\rho$,

$$
\begin{aligned}
|f(z)| & \leq M(\rho, f)^{1 / 2}+2 M(\rho, f)^{1 / 2}\left(M(\rho, f)^{c}+M(\rho, f)^{1 /(4 L)}\right)^{L} \\
& <M(\rho, f)^{3 / 4}\left(1+2^{L+1}\right) \\
& <M(\rho, f),
\end{aligned}
$$

which is a contradiction. Thus $f\left(A\left(\frac{1}{2} \rho, 2 \rho\right)\right)$ covers at least one of the annuli $A\left(R, R^{5}\right)$ or $A\left(\tilde{R}, \tilde{R}^{5}\right)$, and so $f(A(r, 8 r))$ does this as well.

Next, we require a certain Hadamard convexity property.

Lemma 4. Let $f$ be a transcendental meromorphic function with a finite number of poles. Then there exists $R_{1}=R_{1}(f)>0$ such that

$$
M\left(r^{c}, f\right) \geq M(r, f)^{c}, \quad \text { for } r \geq R_{1}, c>1 .
$$

This result follows from the fact that $\log M(r, f)$ is a convex function of $\log r$ such that $\log M(r, f) / \log r \rightarrow \infty$ as $r \rightarrow \infty$, and hence

$$
\frac{r M^{\prime}(r)}{M(r)} \rightarrow \infty \text { as } r \rightarrow \infty
$$

where for definiteness we take $M^{\prime}(r)$ to be the right-derivative; see [33, Lemma 2.2] for a proof of Lemma 4 in the case that $f$ is entire. 
We use Lemma 4 and Harnack's inequality to obtain an annulus covering property in which we assume an opposite type of hypothesis about $m(r, f)$ to that of Lemma 3. (Note that Harnack's inequality is used in a similar way in [21, Lemma 2].)

Lemma 5. Let $f$ be a transcendental meromorphic function with a finite number of poles, let $k>1$, and let $R_{2}=R_{2}(f, k)>0$ be so large that

- $M(r, f)$ is increasing on $\left[R_{2}, \infty\right)$,

- $M(r, f)>r^{k}$ and $s=\sqrt{\log r}>\max \{2 \pi, 4 /(k-1)\}$, for $r>R_{2}$,

- the inequality (2.5) holds for $r \geq R_{2}$ and $c>1$.

If $r>R_{2}$ and

$$
m(\rho, f)>1, \quad \text { for } \rho \in\left(r^{1+1 / s}, r^{k-1 / s}\right),
$$

then the following hold.

(a) We have

$$
\log m(\rho, f) \geq\left(1-\frac{2 \pi}{s}\right) \log M(\rho, f)>0, \quad \text { for } \rho \in\left[r^{1+2 / s}, r^{k-2 / s}\right] .
$$

(b) We have $R>r^{k}$, where $R=M\left(r^{1+2 / s}, f\right)$. Also, if $A \subset A\left(R, R^{k(1-12 / s)}\right)$ is any domain such that $\bar{A}$ is homeomorphic to a closed annulus and surrounds 0 , then $A\left(r^{1+2 / s}, r^{k-2 / s}\right)$ contains a unique component $B$ of $f^{-1}(A)$ with the following properties:

- $\bar{B}$ is homeomorphic to a closed annulus and surrounds 0 ;

- $f(\bar{B})=\bar{A}$;

- $f$ maps the inner and outer boundary components of $B$ onto the corresponding boundary components of $A$.

Proof. By (2.6) the function $u(z)=\log |f(z)|$ is positive harmonic in $A\left(r^{1+1 / s}, r^{k-1 / s}\right)$, so $U(t)=u\left(e^{t}\right)$ is positive harmonic in the strip

$$
S=\{t: \log r+s<\Re(t)<k \log r-s\},
$$

since $s=\sqrt{\log r}$. Now $\log r+2 s<k \log r-2 s$, since $s>4 /(k-1)$. Thus if $t_{1}$ and $t_{2}$ satisfy $\log r+2 s<\Re\left(t_{1}\right)=\Re\left(t_{2}\right)<k \log r-2 s$ and $\left|\Im\left(t_{1}\right)-\Im\left(t_{2}\right)\right| \leq \pi$, then $B\left(t_{1}, s\right) \subset S$ and $\left|t_{2}-t_{1}\right| \leq \pi<s$. So

$$
\frac{s-\pi}{s+\pi} \leq \frac{U\left(t_{2}\right)}{U\left(t_{1}\right)} \leq \frac{s+\pi}{s-\pi},
$$

by Harnack's inequality; see [20, page 35]. Hence, if $z_{1}$ and $z_{2}$ satisfy $r^{1+2 / s}<$ $\left|z_{1}\right|=\left|z_{2}\right|<r^{k-2 / s}$, then

$$
\frac{1-\pi / s}{1+\pi / s} \leq \frac{u\left(z_{2}\right)}{u\left(z_{1}\right)} \leq \frac{1+\pi / s}{1-\pi / s}
$$

Since $\pi / s<1 / 2$, part (a) then follows.

To prove part (b), first note that $R>M(r, f)>r^{k}$. Next

$$
f\left(\left\{z:|z|=r^{1+2 / s}\right\}\right) \subset\left\{z:|z| \leq M\left(r^{1+2 / s}, f\right)\right\}=\{z:|z| \leq R\} .
$$

On the other hand, by part (a),

$$
f\left(\left\{z:|z|=r^{k-2 / s}\right\}\right) \subset\left\{z:|z| \geq M\left(r^{k-2 / s}, f\right)^{1-2 \pi / s}\right\} .
$$


By (2.5), with $c=(k-2 / s) /(1+2 / s)>1$,

$$
\begin{aligned}
M\left(r^{k-2 / s}, f\right)^{1-2 \pi / s} & \geq M\left(r^{1+2 / s}, f\right)^{\frac{k-2 / s}{1+2 / s}(1-2 \pi / s)} \\
& \geq M\left(r^{1+2 / s}, f\right)^{k(1-12 / s)} \\
& =R^{k(1-12 / s)} .
\end{aligned}
$$

Thus, by (2.7) and (2.8),

$$
f\left(\partial A\left(r^{1+2 / s}, r^{k-2 / s}\right)\right) \cap A\left(R, R^{k(1-12 / s)}\right)=\emptyset,
$$

but $f\left(\partial A\left(r^{1+2 / s}, r^{k-2 / s}\right)\right)$ does meet both of the complementary components of $A\left(R, R^{k(1-12 / s)}\right)$. Therefore, since $f$ is analytic in $A\left(r, r^{k}\right)$, we have

$$
f\left(A\left(r^{1+2 / s}, r^{k-2 / s}\right)\right) \supset A\left(R, R^{k(1-12 / s)}\right) .
$$

Now let $A \subset A\left(R, R^{k(1-12 / s)}\right)$ be a domain such that $\bar{A}$ is homeomorphic to a closed annulus and surrounds 0 . Then, by (2.9) and (2.10), the set $f^{-1}(A)$ meets $A\left(r^{1+2 / s}, r^{k-2 / s}\right)$ but does not meet $\partial A\left(r^{1+2 / s}, r^{k-2 / s}\right)$. Thus there is at least one component, $B$ say, of $f^{-1}(A)$ in $A\left(r^{1+2 / s}, r^{k-2 / s}\right)$ and $f(\bar{B})=\bar{A}$. By the argument principle, this component must surround 0 since $f$ is analytic and $f \neq 0$ in $A\left(r^{1+2 / s}, r^{k-2 / s}\right)$. Also, $\bar{B}$ has only one bounded complementary component, namely the one containing 0 , because any other bounded complementary component must lie in $A\left(r^{1+2 / s}, r^{k-2 / s}\right)$ where $f \neq 0$. Hence $\bar{B}$ is homeomorphic to a closed annulus. The mapping property of the two boundary components of $B$ follows from the argument principle since $f: B \rightarrow A$ is a proper map. From this we deduce that $B$ is the unique component of $f^{-1}(A)$ in $A\left(r^{1+2 / s}, r^{k-2 / s}\right)$ with these properties. This proves part (b).

\section{FunCtions With Finitely MANY POLES}

In this section we prove Theorem 1 for functions with finitely many poles. First we deal with a special case.

Lemma 6. Let $f$ be a transcendental meromorphic function with a finite number of poles. Suppose there is a sequence of continua $\Gamma_{m}, m \geq 0$, such that

(1) for each $m \geq 0, \Gamma_{m}$ surrounds 0 and has exactly two complementary components, and $\Gamma_{m}$ is surrounded by $\Gamma_{m+1}$;

(2) $\operatorname{dist}\left(0, \Gamma_{m}\right) \rightarrow \infty$ as $m \rightarrow \infty$;

(3) $f\left(\Gamma_{m}\right)=\Gamma_{m+1}$, for $m \geq 0$.

Then, given any positive sequence $\left(a_{n}\right)$ such that $a_{n} \rightarrow \infty$ as $n \rightarrow \infty$, there exists

$$
\zeta \in I(f) \cap J(f) \quad \text { and } \quad N \in \mathbb{N}
$$

such that (1.5) holds.

Proof. First note that we can assume that $\left(a_{n}\right)$ is an increasing sequence. Also, by renumbering if necessary, we can assume that $f$ has no poles on $\Gamma_{0}$ or outside $\Gamma_{0}$.

By (1) we can define $B_{m}$, for $m \geq 0$, to be the union of $\Gamma_{m}$ and $\Gamma_{m+1}$ and those points that are both outside $\Gamma_{m}$ and inside $\Gamma_{m+1}$. Then $B_{m}$ is a continuum that surrounds 0 and $\partial B_{m}$ is a subset of $\Gamma_{m} \cup \Gamma_{m+1}$. Thus by (3) and the fact that $f$ 
is analytic in a neighbourhood of $B_{m}$, we have, for each $m \geq 0$, exactly one of the following possibilities:

$$
\begin{gathered}
f\left(B_{m}\right)=B_{m+1}, \\
f\left(B_{m}\right)=G_{m+1} \cup B_{m+1},
\end{gathered}
$$

where $G_{m+1}$ is the complementary component of $B_{m+1}$ that contains 0 . Since $J(f)$ is unbounded, we deduce that

$$
B_{m} \cap J(f) \neq \emptyset, \quad \text { for all } m \geq 0 .
$$

If (3.1) holds for all $m \geq M$, say, then (by the hypotheses (1) and (2)) the outside of $B_{M}$ is contained in the Fatou set of $f$, which is impossible. Thus there is a strictly increasing sequence $m(j) \in \mathbb{N}$ such that (3.2) holds for all $m=m(j)$, $j \in \mathbb{N}$. Hence we have the covering properties

$$
f\left(B_{m}\right) \supset B_{m+1}, \quad \text { for } m \geq 0,
$$

and

$$
f\left(B_{m(j)}\right) \supset B_{m(j)}, \quad \text { for } j \in \mathbb{N} .
$$

By (3.5), for any $d \in \mathbb{N}$, we have

$$
f^{d}\left(B_{m(j)}\right) \supset B_{m(j)}, \quad \text { for } j \in \mathbb{N} .
$$

The idea now is to choose a point $\zeta \in B_{0}$ which has an orbit that visits each of the compact sets $B_{m}, m \geq 0$, in order of increasing $m$, except that the orbit remains in each $B_{m(j)}, j \in \mathbb{N}$, for $d(j)$ steps. To arrange this, we introduce a sequence $(p(j))$ of the form

$$
p(j)=d(1)+\cdots+d(j), \quad j \in \mathbb{N},
$$

where $d(j) \in \mathbb{N}$. The sequence $(d(j))$ will be chosen later to give the desired rate of escape of $f^{n}(\zeta)$. We also put $m(0)=0$ and $p(0)=0$.

If we define

$$
E_{n}= \begin{cases}B_{n-p(j-1)}, & \text { for } m(j-1)+p(j-1) \leq n<m(j)+p(j-1), j \in \mathbb{N}, \\ B_{m(j)}, & \text { for } m(j)+p(j-1) \leq n<m(j)+p(j), j \in \mathbb{N},\end{cases}
$$

then it follows from (3.4) and (3.5) that (2.1) holds. Thus, by Lemma 1 and (3.3), there exists a point $\zeta \in E_{0} \cap J(f)=B_{0} \cap J(f)$ such that, for $j \in \mathbb{N}$,

$$
f^{n}(\zeta) \in B_{n-p(j-1)}, \quad \text { for } m(j-1)+p(j-1) \leq n<m(j)+p(j-1),
$$

and

$$
f^{n}(\zeta) \in B_{m(j)}, \quad \text { for } m(j)+p(j-1) \leq n<m(j)+p(j) .
$$

Clearly $\zeta \in I(f) \cap J(f)$ for all possible choices of $p(j)$.

To complete the proof, we choose a subsequence $\left(a_{n(j)}\right)$ of $\left(a_{n}\right)$ such that

$$
B_{m(j)} \subset B\left(0, a_{n(j)}\right),
$$

and then choose $d(j)$ so large that $m(j-1)+p(j-1) \geq n(j)$, for $j \geq 2$. Then, for $j \geq 2$ and $m(j-1)+p(j-1) \leq n<m(j)+p(j)$, the point $f^{n}(\zeta)$ lies inside the outer boundary of $B_{m(j)}$, so

$$
\left|f^{n}(\zeta)\right| \leq a_{n(j)} \leq a_{m(j-1)+p(j-1)} \leq a_{n},
$$

since $\left(a_{n}\right)$ is increasing. This proves (1.5). 
We now prove Theorem 1 for any transcendental meromorphic function with a finite number of poles. Once again we can assume that $\left(a_{n}\right)$ is an increasing sequence such that $a_{n} \rightarrow \infty$ as $n \rightarrow \infty$.

First take $r_{0}$ so large that $r_{0} \geq \max \left\{R_{0}, R_{1}, R_{2}\right\}$, where $R_{0}=R_{0}(f), R_{1}=R_{1}(f)$ and $R_{2}=R_{2}(f, k), 4 \leq k \leq 5$, are the constants appearing in Lemmas 3 , 4 and 5 , and also

$$
r_{0} \geq \exp \left(120^{2}\right)
$$

and

$$
\frac{\log M(r, f)}{\log r} \geq 1000, \quad \text { for } r \geq r_{0} .
$$

Consider the annulus $A_{0}=A\left(r_{0}, r_{0}^{k_{0}}\right)$, where $k_{0}=5$. The first step of the proof is to use Lemma 3 and Lemma 5 to choose a sequence of annuli of the form

$$
A_{m}=A\left(r_{m}, r_{m}^{k_{m}}\right), \quad m \geq 0,
$$

such that, for $m \in \mathbb{N}$,

$$
\begin{gathered}
f\left(A_{m-1}\right) \supset A_{m}, \\
r_{m}>r_{m-1}^{10} \quad \text { and } \quad k_{m} \geq k_{m-1}\left(1-12 / s_{m-1}\right),
\end{gathered}
$$

where $s_{m}=\sqrt{\log r_{m}}$, and

$$
4 \leq k_{m} \leq 5 .
$$

In particular, note that $A_{m}$ surrounds $A_{m-1}$ and $r_{m} \rightarrow \infty$ as $m \rightarrow \infty$.

Suppose that the annuli $A_{0}, A_{1}, \ldots, A_{m-1}, m \in \mathbb{N}$, have been chosen so that they satisfy the above conditions. To choose $A_{m}$ we consider two cases.

Case 1. Suppose first that

$$
\text { there exists } \rho \in\left(3 r_{m-1}, \frac{3}{8} r_{m-1}^{k_{m-1}}\right) \text { such that } m(\rho, f) \leq 1 .
$$

Then

$$
\rho \in\left(\frac{2}{3} \rho, \frac{4}{3} \rho\right) \subset\left(\frac{1}{3} \rho, \frac{8}{3} \rho\right) \subset\left(r_{m-1}, r_{m-1}^{k_{m-1}}\right) .
$$

Since $M(\rho, f) \geq M\left(r_{0}, f\right) \geq 1$, by (3.6) and (3.7), it follows from (3.11) that we can apply Lemma 3 with $r=\frac{1}{3} \rho$. We choose $R$ and $\tilde{R}$ such that

$$
r_{m-1}^{10}<R \quad \text { and } \quad R^{10}<\tilde{R}<M\left(r_{m-1}, f\right)^{1 / 10} \text {; }
$$

this is possible by (3.7). With $r=\frac{1}{3} \rho$, we deduce from (3.11), (3.12) and Lemma 3 that

$$
f\left(A\left(r_{m-1}, r_{m-1}^{k_{m-1}}\right)\right) \text { covers } A\left(R, R^{5}\right) \text { or } A\left(\tilde{R}, \tilde{R}^{5}\right) .
$$

Hence we can choose $r_{m}=R$ or $r_{m}=\tilde{R}$ and $k_{m}=5$ to ensure that (3.8), (3.9) and (3.10) also hold for $A_{m}$.

Case 2. On the other hand, suppose that (3.11) is false; that is,

$$
m(\rho, f)>1, \quad \text { for all } \rho \in\left(3 r_{m-1}, \frac{3}{8} r_{m-1}^{k_{m-1}}\right) .
$$

Then

$$
m(\rho, f)>1, \quad \text { for all } \rho \in\left(r_{m-1}^{1+1 / s_{m-1}}, r_{m-1}^{k_{m-1}-1 / s_{m-1}}\right),
$$

because

$$
\left(1 / s_{m-1}\right) \log r_{m-1}=\sqrt{\log r_{m-1}}>\log 3,
$$


by (3.6). Thus, by Lemma 5 and (3.7),

$$
f\left(A\left(r_{m-1}, r_{m-1}^{k_{m-1}}\right)\right) \supset A\left(R, R^{k_{m-1}\left(1-12 / s_{m-1}\right)}\right),
$$

where $R=M\left(r_{m-1}^{1+2 / s_{m-1}}, f\right)>r_{m-1}^{10}$. Thus we can choose $r_{m}=R$ and $k_{m}=$ $k_{m-1}\left(1-12 / s_{m-1}\right)$ to ensure that (3.8) and (3.9) hold for $A_{m}$. To see that (3.10) also holds for $A_{m}$, note that, for $1 \leq j \leq m-1$ we have, by (3.9),

$$
k_{j} \geq k_{j-1}\left(1-12 / s_{j-1}\right)
$$

and

$$
\frac{s_{j}}{s_{j-1}}=\sqrt{\frac{\log r_{j}}{\log r_{j-1}}} \geq 3 .
$$

Also, $k_{0}=5$ and $s_{0} \geq 120$, by (3.6). Thus

$$
\frac{12}{s_{j}} \leq \frac{1}{10}\left(\frac{1}{3}\right)^{j}, \quad \text { for } 0 \leq j \leq m-1,
$$

so

$$
k_{m} \geq 5 \prod_{j=0}^{m-1}\left(1-\frac{1}{10}\left(\frac{1}{3}\right)^{j}\right) \geq 4
$$

as required.

We have now shown that it is possible to construct a sequence of annuli $A_{m}$ satisfying conditions (3.8), (3.9) and (3.10). Next we use these annuli to obtain a point $\zeta \in I(f) \cap J(f)$ satisfying (1.5).

First suppose that in this process of choosing the annuli we were in Case 2 for all $m \geq M$, say. Without loss of generality we can assume that $M=0$. So, for each $m \in \mathbb{N}$, the annulus $A_{m}$ was obtained by applying Lemma 5 to $A_{m-1}$. We can also deduce from Lemma 5 that if $A \subset A\left(r_{m}, r_{m}^{k_{m}}\right)$ is any domain such that $\bar{A}$ is homeomorphic to a closed annulus which surrounds 0 , then $A_{m-1}$ contains a unique component $B$ of $f^{-1}(A)$ such that $\bar{B}$ is homeomorphic to a closed annulus and surrounds $0, f(\bar{B})=\bar{A}$, and $f$ maps the inner and outer boundary components of $B$ onto the corresponding boundary components of $A$.

Therefore, for each $n \geq m \geq 0$ there is a unique set $\Gamma_{m, n}$, homeomorphic to a closed annulus, contained in $\overline{A_{m}}$ such that

- $f^{n-m}\left(\Gamma_{m, n}\right)=\Gamma_{n, n}=\overline{A_{n}}$, for $n \geq m \geq 0$;

- $\Gamma_{m, n}$ surrounds 0 , for $n \geq m \geq 0$;

- for $n>m \geq 0$, we have $f\left(\Gamma_{m, n}\right)=\Gamma_{m+1, n}$, and $f$ maps the inner and outer boundary components of $\Gamma_{m, n}$ onto the corresponding boundary components of $\Gamma_{m+1, n}$

- $\Gamma_{m, n+1} \subset \Gamma_{m, n}$, for $n \geq m \geq 0$.

Now let $\Gamma_{m}=\bigcap_{n \geq m} \Gamma_{m, n}$, for $m \geq 0$. Then each $\Gamma_{m}, m \geq 0$, is a continuum which surrounds 0 and has two complementary components, and also $\Gamma_{m+1}$ surrounds $\Gamma_{m}$. Moreover, for each $m \geq 0$, we have $f\left(\Gamma_{m}\right)=\Gamma_{m+1}$, since $f\left(\Gamma_{m, n}\right)=\Gamma_{m+1, n}$, for all $n>m$. Thus the sequence of continua $\Gamma_{m}$ has properties (1), (2) and (3) of Lemma 6, so there exists a point $\zeta \in I(f) \cap J(f)$ satisfying (1.5).

The alternative is that in the process of choosing the annuli $A_{m}$ we were in Case 1 infinitely often; that is, we obtained $A_{m}$ by applying Lemma 3 to $A_{m-1}$ for 
infinitely many $m$. When we apply Lemma 3 to $A_{m-1}$, for $m \geq 2$, there exists $\rho$ such that (3.11) holds, and for this value of $\rho$ we have

$$
r_{m-2}^{10}<r_{m-1}<M\left(r_{m-1}, f\right)^{1 / 10}<M\left(\frac{1}{3} \rho, f\right)^{1 / 10},
$$

by (3.9), (3.7) and the fact that $M(r, f)$ is increasing on $\left[r_{0}, \infty\right)$. Thus, by applying Lemma 3 with $r=\frac{1}{3} \rho$ again, but $R=r_{m-2}$ and $\tilde{R}=r_{m-1}$, we obtain

$$
f\left(A_{m-1}\right) \text { covers } A_{m-2} \text { or } A_{m-1} \text {, so } f^{2}\left(A_{m-1}\right) \supset A_{m-1} \text {. }
$$

Hence in this situation, not only do we have

$$
f\left(A_{m-1}\right) \supset A_{m}, \quad \text { for } m \in \mathbb{N},
$$

but, in addition, there is a strictly increasing sequence of positive integers $m(j)$, $j \geq 0$, such that

$$
f\left(A_{m(j)}\right) \text { covers } A_{m(j)-1} \text { or } A_{m(j)} \text {, so } f^{2}\left(A_{m(j)}\right) \supset A_{m(j)}, \text { for } j \in \mathbb{N} \text {. }
$$

We now use similar arguments to those used in the proof of Lemma 6 to prove the existence of a point $\zeta \in I(f)$ satisfying (1.5). In this case, we use (3.13) and (3.14) instead of (3.4) and (3.5). Arguing in this way, we can show that there exists a point $\zeta \in \overline{A_{0}}$ which has an orbit that visits each of the annuli $\overline{A_{m}}, m \geq 0$, in order of increasing $m$, except that after entering $\overline{A_{m(j)}}, j \in \mathbb{N}$, for the first time the orbit remains in $\overline{A_{m(j)-1}} \cup \overline{A_{m(j)}}$ for $d(j)$ steps, ending in $A_{m(j)}$. The only difference from the argument used in Lemma 6 is that here the positive integers $d(j)$ must be even because of (3.14). Clearly $\zeta \in I(f)$ once again, and by choosing the integers $d(j)$ appropriately we can ensure that $\zeta$ satisfies (1.5), as in the proof of Lemma 6.

To complete the proof of Theorem 1 we show that we can take $\zeta \in J(f)$. We do this, using the second statement in Lemma 1 by proving that each of the closed annuli $\overline{A_{m}}$ must meet $J(f)$.

Suppose that $\overline{A_{m(j)}} \subset F(f)$ for some $j \in \mathbb{N}$. Then $\overline{A_{m(j)}} \subset I(f)$ by normality, since $\overline{A_{m(j)}} \cap I(f) \neq \emptyset$. However, by (3.14) and Lemma 11 for each $j \in \mathbb{N}$ there exists a point $z_{j} \in \overline{A_{m(j)}}$ such that the forward orbit of $z_{j}$ remains in $\overline{A_{m(j)-1}} \cup \overline{A_{m(j)}}$, so $z_{j} \notin I(f)$, a contradiction. Therefore our supposition must be false. Hence the sets $\overline{A_{m}}$ meet $J(f)$ for arbitrarily large $m$ and hence for all $m$ by (3.13), as required. This completes the proof of Theorem 1 for functions with finitely many poles.

Remark. Whenever we apply Lemma 3 or (3.2) we have the option to choose the covered annulus to be either large or within a uniformly bounded distance of 0 . If we do this, then the corresponding points of the orbit of $\zeta$ have the same property. Hence we can ensure that (1.5) holds and that

$$
\liminf _{n \rightarrow \infty}\left|f^{n}(\zeta)\right|<\infty \quad \text { and } \quad \limsup _{n \rightarrow \infty}\left|f^{n}(\zeta)\right|=\infty .
$$

\section{Functions With infinitely many poles}

For functions with infinitely many poles, we prove Theorem 1 using the following version of Ahlfors' five islands theorem; see [36, Corollary to Theorem VI.8].

Lemma 7. If $f$ is a transcendental meromorphic function and $D_{i}, 1 \leq i \leq 5$, are simply connected domains bounded by Jordan curves such that the $\overline{D_{i}}$ are disjoint, then for each $R>0$ there are infinitely many domains in $\mathbb{C} \backslash B(0, R)$, each of which is mapped by $f$ univalently onto one of the $D_{i}$. 
Suppose that $f$ is a transcendental meromorphic function with infinitely many poles. We have the following properties:

(1) the image under $f$ of any open disc around a pole of $f$ contains a neighbourhood of $\infty$;

(2) if $D_{0}, D_{1}, D_{2}, D_{3}, D_{4}$ are open discs with disjoint closures and $R>0$, then, by Lemma 7 , there exists a Jordan domain $D$ in $\mathbb{C} \backslash B(0, R)$ such that $f$ maps $D$ univalently onto $D_{i}$, for some $i \in\{0,1, \ldots, 4\}$.

We now obtain a sequence of Jordan domains tending to $\infty$ with certain covering properties. First, we use property (1) and the fact that $f$ has infinitely many poles to obtain open discs

$$
D_{m}=B\left(z_{m}, r_{m}\right), \quad m \geq 0,
$$

such that

$$
\begin{aligned}
& \operatorname{dist}\left(0, D_{m}\right) \rightarrow \infty \text { as } m \rightarrow \infty, \\
& z_{m} \text { is a pole of } f, \quad \text { for } m \geq 0,
\end{aligned}
$$

and

$$
f\left(D_{m}\right) \supset D_{m+1}, \quad \text { for } m \geq 0 .
$$

Next, we use property (2) to obtain Jordan domains $V_{j}, j \geq 0$, such that

$$
\begin{gathered}
\operatorname{dist}\left(0, V_{j}\right) \rightarrow \infty \text { as } j \rightarrow \infty, \\
f\left(D_{5 j+4}\right) \supset V_{j}, \quad \text { for } j \geq 0,
\end{gathered}
$$

and

$$
f\left(V_{j}\right) \supset D_{m(j)}, \quad \text { for } j \geq 0 \text { and some } m(j) \in\{5 j, 5 j+1, \ldots, 5 j+4\} .
$$

By (4.3), (4.5) and (4.6), we have

$$
f^{60}\left(D_{5 j+4}\right) \supset D_{5 j+4}, \quad \text { for } j \geq 0,
$$

since 60 is the least common multiple of $2,3,4,5$ and 6 .

The idea now is to choose a point $\zeta \in \overline{D_{0}}$ which has an orbit that visits each of the sets $\overline{D_{m}}, m \geq 0$, in order of increasing $m$, except that after entering $D_{5 j+4}$, $j \geq 0$, for the first time the orbit remains in $\overline{D_{5 j}} \cup \cdots \cup \overline{D_{5 j+4}} \cup \overline{V_{j}}$ for $d(j)$ steps ending in $\overline{D_{5 j+4}}$.

To arrange this, we introduce a sequence $p(j)$ of the form

$$
p(j)=d(0)+\cdots+d(j), \quad j \geq 0,
$$

where each $d(j) \in 60 \mathbb{N}$, and also put $p(-1)=0$. Then, for $j \geq 0$, define

$$
E_{n}=\overline{D_{n-p(j-1)}}, \quad \text { for } 5 j+p(j-1) \leq n<5 j+5+p(j-1),
$$

and define $E_{n}$, for $5 j+5+p(j-1) \leq n<5 j+5+p(j)$, to be $d(j)$ closed sets, each belonging to $\left\{\overline{D_{5 j}}, \overline{D_{5 j+1}}, \ldots, \overline{D_{5 j+4}}, \overline{V_{j}}\right\}$, which are arranged in the order defined by the covering properties (4.6) and (4.7), starting with $\overline{V_{j}}$ and ending with $\overline{D_{5 j+4}}$. Then (2.1) holds by (4.3) and (4.7), so we can use Lemma 1 to choose the required point $\zeta \in \overline{D_{0}}$ such that, for $j \geq 0$,

$$
f^{n}(\zeta) \in \overline{D_{n-p(j-1)}}, \quad \text { for } 5 j+p(j-1) \leq n<5 j+5+p(j-1),
$$

and

$$
f^{n}(\zeta) \in \overline{D_{5 j}} \cup \cdots \cup \overline{D_{5 j+4}} \cup \overline{V_{j}}, \quad \text { for } 5 j+5+p(j-1) \leq n<5 j+5+p(j) .
$$


Clearly $\zeta \in I(f)$ for all possible choices of $p(j)$ by (4.1) and (4.4). Also, given any positive increasing sequence $\left(a_{n}\right)$ such that $a_{n} \rightarrow \infty$ as $n \rightarrow \infty$, we can choose $d(j)$ appropriately to ensure that $\zeta$ satisfies (1.5), as in the proof of Lemma 6. Finally, note that all poles and their pre-images are in $J(f)$, so we deduce from the second statement of Lemma 1 that $\zeta$ can be taken to lie in $J(f)$, as required. This completes the proof of Theorem 1 .

Remark. Whenever we apply Lemma 7 we have the option to choose the covered disc to be either large or within a uniformly bounded distance of 0 . If we do this, then the corresponding points of the orbit of $\zeta$ have the same property. Hence we can ensure that (1.5) holds and that

$$
\liminf _{n \rightarrow \infty}\left|f^{n}(\zeta)\right|<\infty \quad \text { and } \quad \limsup _{n \rightarrow \infty}\left|f^{n}(\zeta)\right|=\infty .
$$

\section{Proof of Theorem 2}

To prove Theorem 2 we use another annulus covering property related to Bohr's theorem.

Lemma 8. Let $f$ be a transcendental meromorphic function with a finite number of poles, let $c$ and $K$ be positive constants, and let $\alpha, \beta, \gamma, \alpha^{\prime}, \beta^{\prime}, \alpha^{\prime \prime}, \beta^{\prime \prime}$ and $C$ be constants such that

$$
1<\alpha<\beta, \quad 1<\alpha^{\prime}<\beta^{\prime}<\alpha^{\prime \prime}<\beta^{\prime \prime} \leq C \quad \text { and } \quad \alpha^{\prime \prime} / \beta^{\prime} \geq \gamma=\beta / \alpha .
$$

There exists $R_{0}=R_{0}(f, \gamma, C, c, K)>0$ such that if $r>R_{0}$,

$$
1 \leq R \leq K M(r, f) \text { and } m(\sqrt{\alpha \beta} r, f) \leq c \text {, }
$$

then

$$
f(A(\alpha r, \beta r)) \text { covers } A\left(\alpha^{\prime} R, \beta^{\prime} R\right) \text { or } A\left(\alpha^{\prime \prime} R, \beta^{\prime \prime} R\right) \text {. }
$$

Proof. Assume that (5.2) holds for some $r>0$. Then there exists $z_{0}$ such that $\left|z_{0}\right|=\sqrt{\alpha \beta} r$ and $\left|f\left(z_{0}\right)\right| \leq c$. Suppose that $f$ omits in $A(\alpha r, \beta r)$ two values:

$$
w_{1} \in A\left(\alpha^{\prime} R, \beta^{\prime} R\right) \text { and } w_{2} \in A\left(\alpha^{\prime \prime} R, \beta^{\prime \prime} R\right) .
$$

Then $w_{1} \neq w_{2}$ and

$$
g(z)=\frac{f(z)-w_{1}}{w_{2}-w_{1}}
$$

omits in $A(\alpha r, \beta r)$ the values 0 and 1 , so we can apply Lemma 2 to the function $g$. Now

$$
\left|g\left(z_{0}\right)\right| \leq \frac{c+\beta^{\prime} R}{\alpha^{\prime \prime} R-\beta^{\prime} R} \leq \frac{c+1}{\alpha^{\prime \prime} / \beta^{\prime}-1} .
$$

Thus, by Lemma 2 and (5.1), for $|z|=\sqrt{\alpha \beta} r$,

$$
|g(z)| \leq \exp \left(\left(\log ^{+}\left(\frac{c+1}{\gamma-1}\right)+C_{0}\right)\left(\exp \left(\frac{\pi^{2}}{\log \gamma}\right)+1\right)\right)=D,
$$

where $C_{0}$ is a positive absolute constant and the positive constant $D$ depends on $\gamma$ and $c$. Therefore, for $|z|=\sqrt{\alpha \beta} r$, we have

$$
\begin{aligned}
|f(z)| & \leq\left|w_{1}\right|+\left(\left|w_{2}\right|+\left|w_{1}\right|\right)|g(z)| \\
& \leq \beta^{\prime} R+\left(\beta^{\prime \prime} R+\beta^{\prime} R\right) D \\
& \leq(1+2 D) C K M(r, f),
\end{aligned}
$$

by (5.1) and (5.2). 
Now, for any $k>1$ we have

$$
\frac{M(k r, f)}{M(r, f)} \rightarrow \infty \text { as } r \rightarrow \infty
$$

since $\log M(r, f)$ is a convex function of $\log r$ such that $\log M(r, f) / \log r \rightarrow \infty$ as $r \rightarrow \infty$. Therefore, there exists $R_{0}=R_{0}(f, \gamma, C, c, K)$ such that if $r>R_{0}$, then

$$
|f(z)|<M(\sqrt{\alpha \beta} r, f), \quad \text { for }|z|=\sqrt{\alpha \beta} r,
$$

which is a contradiction. Thus we deduce that if $r>R_{0}$, then $f(A(\alpha r, \beta r))$ covers at least one of the annuli $A\left(\alpha^{\prime} R, \beta^{\prime} R\right)$ or $A\left(\alpha^{\prime \prime} R, \beta^{\prime \prime} R\right)$, as required.

We also require the following result due to Zheng [38. (Recall from Section 1 that, for a transcendental meromorphic function with at most finitely many poles, the notions of nested wandering domain and Baker wandering domain are equivalent.)

Lemma 9. Let $f$ be a transcendental meromorphic function with at most finitely many poles. If $f$ has a nested wandering domain $U$, then for a multiply connected domain $A$ in $U$ such that each $f^{n}(A), n \in \mathbb{N}$, contains a closed curve which is not null-homotopic in $U_{n}$, there exist annuli $A_{n}=\left\{z: r_{n}<|z|<R_{n}\right\}, n \in \mathbb{N}$, and $n_{0} \in \mathbb{N}$ such that

$$
A_{n} \subset f^{n}(A), \text { for } n>n_{0},
$$

and $R_{n} / r_{n} \rightarrow \infty$ as $n \rightarrow \infty$.

Proof of Theorem 2. First we assume that $f$ is a transcendental meromorphic function with a finite number of poles and that $c, d$ and $r_{0}$ are positive constants such that $d>1$ and

for all $r \geq r_{0}$ there exists $\rho \in(r, d r)$ such that $m(\rho, f) \leq c$.

Suppose that $\left(a_{n}\right)$ is a positive sequence such that $a_{n} \rightarrow \infty$ as $n \rightarrow \infty$ and $a_{n+1}=O\left(M\left(a_{n}, f\right)\right)$ as $n \rightarrow \infty$. We take a positive constant $K$ and $N \in \mathbb{N}$ so large that

$$
1 \leq a_{n+1} \leq K M\left(a_{n}, f\right) \quad \text { and } \quad a_{n} \geq \max \left\{r_{0}, R_{0}\right\}, \quad \text { for } n \geq N,
$$

where $R_{0}=R_{0}\left(f, d, d^{6}, c, K\right)$ is the constant in Lemma 8. Then, by (5.3), there exist sequences $\left(\rho_{n}^{\prime}\right)$ and $\left(\rho_{n}^{\prime \prime}\right)$ such that, for $n \geq N$,

$$
\rho_{n}^{\prime} \in\left(d a_{n}, d^{2} a_{n}\right) \text { and } m\left(\rho_{n}^{\prime}, f\right) \leq c,
$$

and

$$
\rho_{n}^{\prime \prime} \in\left(d^{4} a_{n}, d^{5} a_{n}\right) \quad \text { and } \quad m\left(\rho_{n}^{\prime \prime}, f\right) \leq c .
$$

Now define, for $n \geq N$,

$$
A_{n}^{\prime}=A\left(d^{-1 / 2} \rho_{n}^{\prime}, d^{1 / 2} \rho_{n}^{\prime}\right) \quad \text { and } \quad A_{n}^{\prime \prime}=A\left(d^{-1 / 2} \rho_{n}^{\prime \prime}, d^{1 / 2} \rho_{n}^{\prime \prime}\right) .
$$

Then

$$
a_{n}<d^{-1 / 2} \rho_{n}^{\prime}<d^{1 / 2} \rho_{n}^{\prime}<d^{-1 / 2} \rho_{n}^{\prime \prime}<d^{1 / 2} \rho_{n}^{\prime \prime}<d^{6} a_{n} .
$$

We now apply Lemma 8 with

$$
r=a_{n}, \alpha r=d^{-1 / 2} \rho_{n}^{\prime}, \beta r=d^{1 / 2} \rho_{n}^{\prime},
$$

and

$$
R=a_{n+1}, \alpha^{\prime} R=d^{-1 / 2} \rho_{n+1}^{\prime}, \beta^{\prime} R=d^{1 / 2} \rho_{n+1}^{\prime}, \alpha^{\prime \prime} R=d^{-1 / 2} \rho_{n+1}^{\prime \prime}, \beta^{\prime \prime} R=d^{1 / 2} \rho_{n+1}^{\prime \prime} .
$$


Then $\alpha^{\prime \prime} / \beta^{\prime}=d^{-1} \rho_{n+1}^{\prime \prime} / \rho_{n+1}^{\prime} \geq d$ by (5.5) and (5.6), and $\beta / \alpha=d$ by (5.8). We deduce from Lemma 8 and (5.4) that for $n \geq N$ we have

$$
f\left(A_{n}^{\prime}\right) \quad \text { covers } A_{n+1}^{\prime} \text { or } A_{n+1}^{\prime \prime}
$$

similarly,

$$
f\left(A_{n}^{\prime \prime}\right) \text { covers } A_{n+1}^{\prime} \text { or } A_{n+1}^{\prime \prime} .
$$

Therefore we can choose, for $n \geq N$, the compact set $E_{n}$ to be either $\overline{A_{n}^{\prime}}$ or $\overline{A_{n}^{\prime \prime}}$ in such a way that

$$
f\left(E_{n}\right) \supset E_{n+1}, \quad \text { for } n \geq N .
$$

Then, by Lemma 1 and (5.7), there exists $\zeta_{N} \in E_{N}$ such that

$$
f^{n-N}\left(\zeta_{N}\right) \in E_{n} \subset \overline{A\left(a_{n}, C a_{n}\right)}, \quad \text { for } n \geq N,
$$

where $C=d^{6}$.

Without loss of generality, we can assume that $\zeta_{N}$ is not a Fatou-exceptional value, so by applying Picard's theorem a finite number of times we can choose $\zeta$ such that $f^{N}(\zeta)=\zeta_{N}$ and $\left|f^{n}(\zeta)\right| \geq a_{n}$, for $n=1, \ldots, N-1$. Thus, for this $\zeta$ we have (possibly with a larger constant $C$ )

$$
a_{n} \leq\left|f^{n}(\zeta)\right| \leq C a_{n}, \quad \text { for } n \in \mathbb{N} .
$$

To ensure that we also have $\zeta \in J(f)$, we observe that $f$ cannot have a nested wandering domain $U$. For this would imply, by Lemma 9 , the existence of a sequence of annuli $A\left(r_{n}, R_{n}\right)$, where $r_{n}<R_{n}$, such that $r_{n} \rightarrow \infty$ as $n \rightarrow \infty$ and $R_{n} / r_{n} \rightarrow \infty$ as $n \rightarrow \infty$, and for $n$ large enough

$$
\overline{A\left(r_{n}, R_{n}\right)} \subset f^{n}(U) .
$$

Thus $m(r, f) \rightarrow \infty$ as $r \rightarrow \infty$ through

$$
\bigcup_{n=1}^{\infty}\left(r_{n}, R_{n}\right)
$$

contrary to hypothesis (1.7). It follows that $J(f)$ has an unbounded component, by [37, Theorem 1], for example. Thus, by (5.9), we can choose $\zeta_{N} \in E_{N} \cap J(f)$, as required.

In the other direction, if there do not exist positive constants $c, d$ and $r_{0}$ such that $d>1$ and (1.7) holds, then there exists a sequence of annuli $A\left(r_{n}, R_{n}\right)$, where $0<r_{n}<R_{n}$, such that $r_{n} \rightarrow \infty$ as $n \rightarrow \infty, R_{n} / r_{n} \rightarrow \infty$ as $n \rightarrow \infty$ and

$$
m(r, f)>1, \quad \text { for } r_{n}<r<R_{n}, n \in \mathbb{N} .
$$

Then by an argument similar to that in the proof of Lemma 5 we deduce that

$$
m(r, f)>M(r, f)^{\delta}, \quad \text { for } 2 r_{n}<r<\frac{1}{2} R_{n}, n \geq N,
$$

for some constant $0<\delta<1$ and some $N \in \mathbb{N}$. Since $M(r, f)^{\delta} / r \rightarrow \infty$ as $r \rightarrow \infty$, it is not possible to satisfy (1.6) for any positive sequence $\left(a_{n}\right)$ having the property that: $a_{n(j)+1}=O\left(a_{n(j)}\right)$ as $j \rightarrow \infty$, for a sequence $n(j), j \in \mathbb{N}$, such that $a_{n(j)} \sim$ $\sqrt{r_{j} R_{j}}$ as $j \rightarrow \infty$. This completes the proof of Theorem 2, 


\section{Proof of Theorem 3}

To prove Theorem 3 we need several preliminary results. First we give a lemma based on two key ideas from [15, proof of Theorem 1].

Lemma 10. Let $f$ be a transcendental meromorphic function and let $E \subset \mathbb{C}$ be a non-empty set.

(a) If $E$ has a subset $E^{\prime}$ with at least 3 points, such that $E^{\prime}$ is backwards invariant under $f$, and int $E \cap J(f)=\emptyset$, then $J(f) \subset \partial E^{\prime}$ and $J(f) \subset \partial E$.

(b) If $z \in \partial E \backslash J(f)$, then $z$ lies in a Fatou component of $f$ which meets both $E$ and $E^{c}$. In particular, if every component of $F(f)$ that meets $E$ is contained in $E$, then $\partial E \subset J(f)$.

Proof. Since $E^{\prime}$ is backwards invariant under $f$ and $f$ is an open map, we have $f\left(\mathbb{C} \backslash \overline{E^{\prime}}\right) \subset \mathbb{C} \backslash \overline{E^{\prime}}$. Thus $\left(f^{n}\right)$ forms a normal family in $\mathbb{C} \backslash \overline{E^{\prime}}$ by Montel's theorem, so $J(f) \subset \overline{E^{\prime}} \subset \bar{E}$. Since int $E^{\prime} \subset \operatorname{int} E \subset F(f)$, we deduce that $J(f) \subset \partial E^{\prime}$ and $J(f) \subset \partial E$.

Part (b) follows immediately from the fact that any open disc centred at a point of $\partial E$ meets both $E$ and $E^{c}$.

Proving that the hypotheses of Lemma 10(a) hold for the sets $L(f), M(f)$ and $I^{a}(f)$ will be straightforward. However, to apply Lemma 10(b) to these sets we must determine which Fatou components of $f$ can meet them and also meet their complements. To do this we use the following distortion lemma, which is obtained by combining [7, Lemma 7] and [28, Theorem 1].

Lemma 11. Let $G$ be an unbounded open set in $\mathbb{C}$ such that $\partial G$ has at least two finite points, and let $f$ be analytic in $G$. Let $D$ be a domain contained in $G$ such that $f^{n}(D) \subset G$, for $n \geq 1$, and $f^{n}(z) \rightarrow \infty$ as $n \rightarrow \infty$, for $z \in D$.

(a) For any compact disc $\Delta \subset D$, there exist $C>1$ and $n_{0} \in \mathbb{N}$ such that

$$
\left|f^{n}\left(z^{\prime}\right)\right| \leq\left|f^{n}(z)\right|^{C}, \quad \text { for } z, z^{\prime} \in \Delta, n \geq n_{0} .
$$

(b) If, in addition, one of the following holds:

(i) $\hat{\mathbb{C}} \backslash G$ contains an unbounded connected set, or

(ii) $D=G$ and $f$ does not extend analytically to $\infty$, then for any compact disc $\Delta \subset D$, there exist $C>1$ and $n_{0} \in \mathbb{N}$ such that

$$
\left|f^{n}\left(z^{\prime}\right)\right| \leq C\left|f^{n}(z)\right|, \quad \text { for } z, z^{\prime} \in \Delta, n \geq n_{0} .
$$

We also need the following topological lemma. Here, for a set $E \subset \mathbb{C}$, the set $\widetilde{E}$ denotes the union of $E$ and its bounded complementary components.

Lemma 12. Let $V \subset \mathbb{C}$ be an open set with components $V_{n}, n \in \mathbb{N}$. For $n \in \mathbb{N}$, put

$$
\Omega_{V_{n}}=\bigcup\left\{\widetilde{V_{m}}: m \in \mathbb{N}, m \neq n, \widetilde{V_{n}} \subset \widetilde{V_{m}}\right\} .
$$

Then the following cases can arise:

(1) $\Omega_{V_{N}}=\mathbb{C}=\widetilde{V_{N}}$ for some $N \in \mathbb{N}$;

(2) $\Omega_{V_{N}}=\mathbb{C}$ for some $N \in \mathbb{N}$ but each $V_{n}, n \in \mathbb{N}$, is bounded, so there is a sequence $\left(n_{j}\right)$ in $\mathbb{N}$ such that

$$
\widetilde{V_{n_{1}}} \subset \widetilde{V_{n_{2}}} \subset \cdots \text { and } \bigcup_{j \geq 1} \widetilde{V_{n_{j}}}=\mathbb{C} ;
$$


(3) $\Omega_{V_{N}}$ is unbounded for some $N \in \mathbb{N}$ and $\Omega_{V_{N}} \neq \mathbb{C}$;

(4) $\Omega_{V_{n}}$ is bounded for all $n \in \mathbb{N}$.

This lemma can be found in 27, Lemma 3]. There the proof is given for the case when $V=\{z: u(z)>M\}$, where $u$ is a real-valued, continuous function defined in $\mathbb{C}$ and $M \in \mathbb{R}$, but this proof applies without change when $V$ is an arbitrary open set. Note that each set $\Omega_{V_{n}}$ is full; that is, it has no bounded complementary components.

Using Lemmas 11 and 12 we obtain the following result about the behaviour of the iterates of a transcendental meromorphic function in its escaping Fatou components; this result may be of independent interest. Later in the section, we give examples to show that the hypothesis that $U$ is not a quasi-nested wandering domain cannot be omitted in this result.

Theorem 5. Let $f$ be a transcendental meromorphic function and let $U$ be a component of $F(f)$ which is contained in $I(f)$. If $U$ is not a quasi-nested wandering domain, then the estimate (6.2) holds for any compact disc $\Delta \subset U$.

Proof. For $n \in \mathbb{N}$, let $U_{n}$ be the Fatou component of $f$ such that $f^{n}(U) \subset U_{n}$. By Lemma 12, applied to the open set $V=\bigcup_{n \in \mathbb{N}} U_{n}$, the following cases can arise:

(1) $\Omega_{U_{N}}=\mathbb{C}=\widetilde{U_{N}}$ for some $N \in \mathbb{N}$;

(2) $\Omega_{U_{N}}=\mathbb{C}$ for some $N \in \mathbb{N}$ but each $U_{n}, n \in \mathbb{N}$, is bounded, so there is a sequence $\left(n_{j}\right)$ in $\mathbb{N}$ such that

$$
\widetilde{U_{n_{1}}} \subset \widetilde{U_{n_{2}}} \subset \cdots \text { and } \bigcup_{j \geq 1} \widetilde{U_{n_{j}}}=\mathbb{C} ;
$$

(3) $\Omega_{U_{N}}$ is unbounded for some $N \in \mathbb{N}$ and $\Omega_{U_{N}} \neq \mathbb{C}$;

(4) $\Omega_{U_{n}}$ is bounded for all $n \in \mathbb{N}$.

In case (1), either $U_{N}$ is periodic, in which case $U_{N}$ is an invariant Baker domain because $U_{N} \subset I(f)$, or all the Fatou components $U_{n}, n>N$, are contained in bounded complementary components of $U_{N}$. If $U_{N}$ is an invariant Baker domain, then Lemma 11(b)(ii) can be applied to $f$ in $G=U_{N}$, and hence (6.2) holds for any compact disc $\Delta \subset U$. On the other hand, if all the Fatou components $U_{n}, n>N$, are contained in bounded complementary components of $U_{N}$, then Lemma 11(b)(i) can be applied to $f$ in the open set $G=\bigcup_{n>N} U_{n}$, and hence (6.2) holds for any compact disc $\Delta \subset U$.

In case (3), the boundary of $\Omega_{U_{N}}$ has an unbounded component which is contained in an unbounded complementary component of the set

$$
G=\bigcup_{n \in \mathbb{N}} U_{n} .
$$

Since $G$ is invariant under $f$, we can apply Lemma 11(b)(i) to $f$ in $G$ with $D=U_{1}$ to deduce that (6.2) holds for any compact disc $\Delta \subset U$.

In case (4), the complement of the set

$$
\bigcup_{n \in \mathbb{N}} \Omega_{U_{n}}
$$

is connected and unbounded and lies in the complement of the set $G$ defined in (6.4), so we can apply Lemma 11(b)(i) again to $f$ in $G$ with $D=U_{1}$.

Thus the estimate (6.2) holds for any compact disc $\Delta \subset U$ unless case (2) holds. In this case $U$ is a quasi-nested wandering domain, as required. 
We now use Theorem [5 and Lemma 11 to prove the following result. This indicates when Lemma 10(b) can be applied if $E$ is $L(f), M(f)$ or $I^{a}(f)$.

Lemma 13. Let $f$ be a transcendental meromorphic function, let $U$ be a component of $F(f)$ and let $a=\left(a_{n}\right)$ be a positive sequence with $a_{n} \rightarrow \infty$ as $n \rightarrow \infty$.

(a) If $U \cap L(f) \neq \emptyset$, then $U \subset L(f)$.

(b) If $U \cap M(f) \neq \emptyset$, then $U \subset M(f)$.

(c) If $U \cap I^{a}(f) \neq \emptyset$ and $U$ is not a quasi-nested wandering domain, then $U \subset I^{a}(f)$.

Proof. First recall that if $U \cap I(f) \neq \emptyset$, then $U \subset I(f)$.

To prove part (a), take $z \in U \cap L(f)$. Then

$$
\limsup _{n \rightarrow \infty} \frac{1}{n} \log \left|f^{n}(z)\right|<\infty .
$$

Let $\Delta$ be any compact disc in $U$ with centre $z$. Then, by (6.1), there exists $C>1$ such that

$$
\limsup _{n \rightarrow \infty} \frac{1}{n} \log \left|f^{n}\left(z^{\prime}\right)\right| \leq C \limsup _{n \rightarrow \infty} \frac{1}{n} \log \left|f^{n}(z)\right|<\infty, \quad \text { for } z^{\prime} \in \Delta,
$$

so $\Delta \subset L(f)$. Hence $U \subset L(f)$. A similar argument applies to $M(f)$.

To prove part (c), take $z \in U \cap I^{a}(f)$. Then

$$
\left|f^{n}(z)\right|=O\left(a_{n}\right) \text { as } n \rightarrow \infty .
$$

Since $U$ is not a quasi-nested wandering domain we deduce by Theorem 5 that if $\Delta$ is any compact disc in $U$ with centre $z$, then there exist $C>1$ and $n_{0} \in \mathbb{N}$ such that

$$
\left|f^{n}\left(z^{\prime}\right)\right| \leq C\left|f^{n}(z)\right|, \quad \text { for } z^{\prime} \in \Delta, n \geq n_{0},
$$

so $\Delta \subset I^{a}(f)$ by (6.5). Hence $U \subset I^{a}(f)$, as required.

Proof of Theorem 13. Let $a=\left(a_{n}\right)$ be a positive sequence such that $a_{n} \rightarrow \infty$ as $n \rightarrow \infty$. We consider a positive sequence $a^{\prime}=\left(a_{n}^{\prime}\right)$ such that

$$
a_{n}^{\prime} \leq a_{n} \text { and } a_{n}^{\prime} \text { is increasing, for } n \in \mathbb{N}, \quad \text { and } \quad a_{n}^{\prime} \rightarrow \infty \text { as } n \rightarrow \infty .
$$

It is clear from the definitions that $I^{a^{\prime}}(f) \subset I^{a}(f)$, that $I^{a^{\prime}}(f)$ is backwards invariant under $f$, and that each of the sets $L(f)$ and $M(f)$ is completely invariant under $f$. Also, each of the sets

$$
L(f) \cap J(f), M(f) \cap J(f) \text { and } I^{a^{\prime}}(f) \cap J(f)
$$

is non-empty, by Theorem 1 .

Therefore

- each of $L(f) \cap J(f), M(f) \cap J(f)$ and $I^{a^{\prime}}(f) \cap J(f)$ is a non-empty backwards invariant subset of $I(f) \cap J(f)$ and so is infinite, since for every $\zeta$ at least one of $\zeta, f(\zeta)$ or $f^{2}(\zeta)$ is not Fatou-exceptional and so has an infinite backwards orbit;

- each of $L(f), M(f)$ and $I^{a}(f)$ contains no periodic points, and so has no interior points which lie in $J(f)$.

Thus, by Lemma 10(a), $L(f), M(f)$ and $I^{a}(f)$ are each dense in $J(f)$, and

$$
J(f) \subset \partial L(f), \quad J(f) \subset \partial M(f) \text { and } J(f) \subset \partial I^{a}(f) .
$$


By Lemma 13(a) and (b), and Lemma 10(b), we have

$\partial L(f) \subset J(f)$ and $\partial M(f) \subset J(f)$, so $J(f)=\partial L(f)$ and $J(f)=\partial M(f)$.

This proves part (c).

Part (d) follows immediately from (6.6), Lemma 13(c) and Lemma 10(b). This completes the proof of Theorem 3 .

We now show that we cannot strengthen the statement of Theorem 3 (d) or Corollary 1(a) to assert that we always have $J(f)=\partial I^{a}(f)$. More precisely, we show that for a meromorphic function $f$ with a finite number of poles, if $U$ is a nested wandering domain, then there exists a sequence $\left(a_{n}\right)$ for which $\partial I^{a}(f) \cap U \neq \emptyset$, and hence $\partial I^{a}(f) \not \subset J(f)$. (Recall from Section 1 that, for such a meromorphic function, any quasi-nested wandering domain is a nested wandering domain.) This theorem also shows that the conclusion of Theorem 5 fails if $U$ is a nested wandering domain of a transcendental meromorphic function with a finite number of poles.

Theorem 6. Let $f$ be a transcendental meromorphic function with a finite number of poles and with a nested wandering domain $U$. Then there exist points $z_{0}, z_{0}^{\prime} \in U$ such that

$$
\left|\frac{f^{n}\left(z_{0}^{\prime}\right)}{f^{n}\left(z_{0}\right)}\right| \rightarrow \infty \text { as } n \rightarrow \infty \text {. }
$$

Thus, if $a_{n}=\left|f^{n}\left(z_{0}\right)\right|, n \in \mathbb{N}$, then $z_{0} \in I^{a}(f)$ but $z_{0}^{\prime} \notin I^{a}(f)$, so $\partial I^{a}(f) \cap U \neq \emptyset$.

Proof. First note that it is sufficient to construct the required points $z_{0}$ and $z_{0}^{\prime}$ in $f^{N}(U)$, where $N \geq 0$.

Since $f$ has a finite number of poles, it has a direct tract. Thus, by using a method of Eremenko (see [15, Theorem 1] and [12, proof of Theorem 3.1]), we can show that in any annulus $A\left(\frac{1}{2} r, 2 r\right)$, where $r$ is large enough, there exists $z_{0}^{\prime}$ such that

$$
\left|f^{n+1}\left(z_{0}^{\prime}\right)\right| \geq \frac{1}{2} M\left(\left|f^{n}\left(z_{0}^{\prime}\right)\right|, f\right), \quad \text { for } n \geq 0 .
$$

Next, by Lemma 9, there exists a sequence of annuli $A\left(r_{n}, R_{n}\right)$, where $0<r_{n}<R_{n}$, such that $r_{n} \rightarrow \infty$ as $n \rightarrow \infty$ and $R_{n} / r_{n} \rightarrow \infty$ as $n \rightarrow \infty$, and for $n$ large enough

$$
\overline{A\left(r_{n}, R_{n}\right)} \subset f^{n}(U) .
$$

Thus we can choose $N \in \mathbb{N}$ so large that, with $\rho_{N}=\sqrt{r_{N} R_{N}}$,

$$
\begin{gathered}
\text { there exists } z_{0}^{\prime} \in A\left(2 \rho_{N}, R_{N}\right) \subset f^{N}(U) \text { such that (6.7) holds, } \\
\text { (6.8) holds for } n \geq N,
\end{gathered}
$$

and also, for all $r^{\prime}>r \geq r_{N}$,

$$
M(r, f)>r
$$

and

$$
\frac{M\left(r^{\prime}, f\right)}{M(r, f)} \geq\left(\frac{r^{\prime}}{r}\right)^{2} ; \quad \text { in particular, } \quad M(2 r, f) \geq 4 M(r, f) .
$$

The estimate (6.10) is a special case of Lemma 4, or it follows directly from the convexity of $\log M(r, f)$ with respect to $\log r$.

Using (6.7), the fact that $\left|z_{0}^{\prime}\right|>2 \rho_{N}$, and the second estimate in (6.10), we deduce by induction that

$$
\left|f^{n}\left(z_{0}^{\prime}\right)\right| \geq 2 M^{n}\left(\rho_{N}, f\right), \quad \text { for } n \in \mathbb{N} .
$$


On the other hand, if $\left|z_{0}\right|=r_{N}$, then $z_{0} \in f^{N}(U)$ and

$$
\left|f^{n}\left(z_{0}\right)\right| \leq M^{n}\left(r_{N}, f\right), \quad \text { for } n \in \mathbb{N} \text {. }
$$

Hence, by (6.9) and the first estimate in (6.10), we deduce by induction that

$$
\frac{\left|f^{n}\left(z_{0}^{\prime}\right)\right|}{\left|f^{n}\left(z_{0}\right)\right|} \geq \frac{2 M^{n}\left(\rho_{N}, f\right)}{M^{n}\left(r_{N}, f\right)} \geq 2\left(\frac{\rho_{N}}{r_{N}}\right)^{2^{n}} \rightarrow \infty \text { as } n \rightarrow \infty,
$$

as required.

In Theorem [6] the sequence $a_{n}=\left|f^{n}\left(z_{0}\right)\right|$ tends to $\infty$ quickly; for example, $z_{0} \in Z(f)$. Our next example shows that

- in the absence of a direct tract, $J(f)=\partial I^{a}(f)$ need not hold even if $a_{n}$ tends to $\infty$ at a much slower rate;

- in Corollary 1(a), the assumption about the existence of a direct tract cannot be omitted.

This example also shows that the conclusion of Theorem 5 can fail for a quasi-nested wandering domain $U$ that is not a Baker wandering domain.

Example 1. There is a positive increasing sequence $a=\left(a_{n}\right)$ such that $a_{n} \rightarrow \infty$ as $n \rightarrow \infty$ and $a_{n+1}=O\left(a_{n}\right)$ as $n \rightarrow \infty$, and a transcendental meromorphic function $f$ with a Fatou component $U$ containing $z_{0}$ and $z_{0}^{\prime}$ such that

(a) $z_{0} \in I^{a}(f)$ but $z_{0}^{\prime} \notin I^{a}(f)$, so $\partial I^{a}(f) \cap U \neq \emptyset$;

(b) $\limsup _{n \rightarrow \infty}\left|f^{n}\left(z_{0}^{\prime}\right) / f^{n}\left(z_{0}\right)\right|=\infty$;

(c) $U$ is a quasi-nested wandering domain but not a Baker wandering domain.

Proof. We take $a, b, z_{0}$ and $z_{0}^{\prime}$ such that

$$
1<a<z_{0}<z_{0}^{\prime}<b, \quad a^{2}>4 b, \quad\left(z_{0}+1\right)^{4}>b^{3} \quad \text { and } \quad z_{0}^{\prime}>z_{0}+3 .
$$

The sequence $\left(a_{n}\right)$ is defined as $a_{n}=b^{n+1}, n \geq 0$.

To construct $f$, we define a sequence of closed annuli $B_{n}$ as follows. Here we use the notation

$$
B(z ; r, R)=\{w: r \leq|w-z| \leq R\}, \quad \text { for } z \in \mathbb{C}, 0<r<R .
$$

First we choose a sequence $\left(n_{k}\right)$ in $\{0,1,2, \ldots\}$ such that

$$
b^{n_{k}}<\left(z_{0}+1\right)^{2^{k}} \leq b^{n_{k}+1}, \quad \text { for } k \geq 0 .
$$

Then $n_{0}=0, n_{1}=1$ and $n_{2}=3$, by (6.11). Also,

$$
n_{k+1}+1>2 n_{k}, \text { for } k \geq 0, \quad \text { so } n_{k+1} \geq n_{k}+2 \text {, for } k \geq 1 \text {, }
$$

and we define the corresponding subsequence of closed annuli

$$
B_{n_{k}}=B\left(0 ; a^{2^{k}}, b^{2^{k}}\right), \quad k \geq 0 .
$$

Now we define the rest of the $B_{n}$ to be certain nested finite sequences of closed annuli lying between adjacent annuli $B_{n_{k}}$. For $k \geq 1$, we put

$$
p_{k}=\frac{1}{2} a^{2^{k}}, \quad \text { so } \quad p_{k}>2 b^{2^{k-1}},
$$

by (6.11),

$$
m_{k}=n_{k+1}-n_{k}-1, \text { for } k \geq 0, \text { so } m_{k} \geq 1, \text { for } k \geq 1,
$$


by (6.13), and choose $t_{k}, 0<t_{k}<1$, such that

$$
\frac{t_{k}}{a^{2^{k}}}<\frac{1}{2 b^{2^{k}}} \text {. }
$$

Then define

$$
B_{n_{k}+j}=B\left(p_{k} ; \frac{t_{k}^{j-1}}{b^{2^{k}}}, \frac{t_{k}^{j-1}}{a^{2^{k}}}\right), \quad j=1, \ldots, m_{k},
$$

which are $m_{k}$ nested disjoint closed annuli lying between $B_{n_{k-1}}$ and $B_{n_{k}}$. The function

$$
z \mapsto p_{k}+\frac{1}{z}, \quad z \in B_{n_{k}}, k \geq 1,
$$

maps $B_{n_{k}}$ one-to-one onto $B_{n_{k}+1}$, and the function

$$
z \mapsto p_{k}+t_{k}\left(z-p_{k}\right)
$$

maps $B_{n_{k}+j}$ one-to-one onto $B_{n_{k}+j+1}$, for $j=1, \ldots, m_{k}-1$. Note that the annuli $B_{n_{k}}, B_{n_{k}+1}, \ldots, B_{n_{k}+m_{k}}$ all have modulus $2^{k} \log (b / a)$.

Finally, for $k \geq 1$, we put $S_{k}=t_{k}^{2\left(m_{k}-1\right)}$ so that the function

$$
z \mapsto \frac{S_{k}}{\left(z-p_{k}\right)^{2}}
$$

maps $B_{n_{k}+m_{k}}$ two-to-one onto $B_{n_{k+1}}=B\left(0 ; a^{2^{k+1}}, b^{2^{k+1}}\right)$.

Now we use (6.15), 6.16) and (6.17) to define a function $g$ on the annuli $B_{n}$, $n \geq 1$, and we also set $g(z)=z^{2}$ on $B_{0}$.

We have

$$
g^{n}\left(B_{0}\right)=B_{n}, \quad \text { for } n \geq 1, \quad \text { and } \quad g^{n_{k}}(z)=z^{2^{k}}, \quad \text { for } k \geq 0,
$$

since

$$
g^{m_{k}+1}(z)=z^{2}, \quad \text { for } z \in B_{n_{k}}, k \geq 0 .
$$

Next, for $n \geq 0$, we choose closed annuli $B_{n}^{\prime} \subset B_{n}$, with $B_{n}^{\prime}$ and $B_{n}$ concentric, and $\partial B_{n}^{\prime}$ so close to $\partial B_{n}$ that, for $n \geq 0$,

$$
z_{0}, z_{0}^{\prime} \in B_{0}^{\prime}, \quad g\left(B_{n}^{\prime}\right) \subset B_{n+1}^{\prime} \text { and } \operatorname{dist}\left(\partial g\left(B_{n}^{\prime}\right), \partial B_{n+1}^{\prime}\right)>0 .
$$

Now, for $k \geq 0$, let

$$
E_{k}=\bigcup_{n=n_{k}}^{n_{k}+m_{k}} B_{n}
$$

and let $P_{k}$ be a finite set, including $\infty$, with one point in each component of $\hat{\mathbb{C}} \backslash\left(E_{k} \cup\left\{z:|z| \leq b^{2^{k-1}}\right\}\right)$. Note that $E_{k} \cap\left\{z:|z| \leq b^{2^{k-1}}\right\}=\emptyset$ and $g$ is analytic on each set $E_{k}$.

For each $k \geq 0$, we can use Runge's theorem (see [18]) to choose a rational function $f_{k}$ with poles in the set $P_{k}$ such that

$$
\sup _{E_{k}}\left|f_{0}+f_{1}+\cdots+f_{k}-g\right|<\varepsilon_{k} \quad \text { and } \sup \left\{\left|f_{k}(z)\right|:|z| \leq b^{2^{k-1}}\right\}<\varepsilon_{k},
$$

where the positive sequence $\varepsilon_{k}, k \geq 0$, is so small that

$$
f(z)=f_{0}(z)+f_{1}(z)+\cdots \quad \text { is locally uniformly convergent on } \mathbb{C},
$$


and (using (6.19) ) the function $f$ is so close to $g$ on the annuli $B_{n}, n \geq 0$, that

$$
f\left(B_{n}^{\prime}\right) \subset B_{n+1}^{\prime}, \quad \text { for } n \geq 0, \quad \text { so } \bigcup_{n=0}^{\infty} B_{n}^{\prime} \subset F(f) \cap I(f),
$$

by Montel's theorem, and also, by (6.18), that

$$
\left|f^{m_{k}+1}(z)-g^{m_{k}+1}(z)\right|=\left|f^{m_{k}+1}(z)-z^{2}\right|<1, \text { for } z \in B_{n_{k}}^{\prime}, k \geq 0 .
$$

Hence

$$
f^{n_{k}}(z) \in B_{n_{k}}^{\prime} \text { and }\left|f^{n_{k+1}}(z)-f^{n_{k}}(z)^{2}\right|<1 \text {, for } z \in B_{0}^{\prime}, k \geq 0 \text {. }
$$

By (6.19) and (6.21) we see that $z_{0}$ and $z_{0}^{\prime}$ lie in the same Fatou component of $f$, say $U$. We now show that $z_{0} \in U \cap I^{a}(f)$, where $a_{n}=b^{n+1}, n \geq 0$. Let $X_{k}=\left|f^{n_{k}}\left(z_{0}\right)\right|, \quad k \geq 0$. Then $X_{0}=z_{0}>1$ and, by (6.19) and (6.22),

$$
X_{k+1} \leq X_{k}^{2}+1, \quad \text { for } k \geq 0 .
$$

Thus by induction

$$
X_{k} \leq\left(X_{0}+1\right)^{2^{k}}-1, \quad \text { for } k \geq 0 .
$$

Therefore, for $k \geq 0$ and $j=0,1, \ldots, m_{k}$, by (6.12),

$$
\left|f^{n_{k}+j}\left(z_{0}\right)\right| \leq\left(X_{0}+1\right)^{2^{k}}-1 \leq b^{n_{k}+1} \leq b^{n_{k}+j+1},
$$

so $z_{0} \in I^{a}(f)$, as required.

Now let $Y_{k}=\left|f^{n_{k}}\left(z_{0}^{\prime}\right)\right|, k \geq 0$. Then $Y_{0}=z_{0}^{\prime}>1$ and, by (6.19) and (6.22),

$$
Y_{k+1} \geq Y_{k}^{2}-1, \quad \text { for } k \geq 0 \text {. }
$$

It again follows by induction that

$$
Y_{k} \geq\left(Y_{0}-1\right)^{2^{k}}+1, \quad \text { for } k \geq 0 .
$$

Therefore, for $k \geq 0$, by (6.11) and (6.12),

$$
\left|f^{n_{k}}\left(z_{0}^{\prime}\right)\right| \geq\left(z_{0}^{\prime}-1\right)^{2^{k}}+1>\left(z_{0}+2\right)^{2^{k}}>\left(\frac{z_{0}+2}{z_{0}+1}\right)^{2^{k}} b^{n_{k}}=\left(\frac{z_{0}+2}{z_{0}+1}\right)^{2^{k}} \frac{a_{n_{k}}}{b},
$$

so $z_{0}^{\prime} \notin I^{a}(f)$. This proves part (a).

By Theorem [3(d), the Fatou component $U$ must be a quasi-nested wandering domain. Thus the Fatou components $U_{n} \supset B_{n}^{\prime}, n \geq 0$, are disjoint. Since $m_{k} \geq 2$ for $k \geq 3$, by (6.13), there are infinitely many of these Fatou components that do not surround 0 , so $U$ is not a Baker wandering domain.

Remark. The proof of Example 1 can easily be modified to give the same type of example in which $\left(a_{n}\right)$ is any positive increasing sequence such that $a_{n} \rightarrow \infty$ as $n \rightarrow \infty$ and $a_{n+1}=O\left(a_{n}\right)$ as $n \rightarrow \infty$.

\section{Proof of Theorem 4}

Theorem 4 follows easily from Theorem 3 , Corollary 1 and the definition of $M(f)$.

Proof of Theorem 4. In part (a), $f$ has a direct tract and no nested wandering domains. Hence

$$
J(f)=\partial L(f)=\partial M(f)=\partial I^{a}(f),
$$

by Theorem 3(c) and Corollary 1(a). In this situation we know that $J(f)$ has at least one unbounded component; see [12, Theorem 5.3]. Hence the sets $\partial L(f)$, $\partial M(f)$ and $\partial I^{a}(f)$ each have at least one unbounded component, as required. 
To prove part (a)(ii), note that if $f$ is entire and $J(f)$ has a bounded component, then $f$ has a multiply connected Fatou component [23, Theorem 1] and so has a nested wandering domain, by [2, Theorem 3.1]. Thus all the components of $J(f)$ are unbounded, so this is also true for all the components of $\partial L(f), \partial M(f)$ and $\partial I^{a}(f)$, as required.

Theorem 4(b) follows immediately from the fact that if $f$ has a direct tract, then any nested wandering domain lies in $Z(f)$, as mentioned in the introduction before Corollary 1, together with the fact that $M(f) \cap Z(f)=\emptyset$.

\section{EXAMPLES}

We end the paper by giving a number of explicit examples to show how varied the structures of the sets $L(f), M(f)$ and $I^{a}(f)$ can be. Here, as usual, $a=\left(a_{n}\right)$ is a positive sequence such that $a_{n} \rightarrow \infty$ as $n \rightarrow \infty$.

Example 2. Let

$$
f(z)=\lambda e^{z}, \quad \text { where } 0<\lambda<1 / e .
$$

Then the components of $M(f)$ are all singletons, and hence so are those of $L(f)$ and also $I^{a}(f)$ when $I^{a}(f) \subset M(f)$. However, all the components of $\overline{L(f)}, \overline{M(f)}$ and $\overline{I^{a}(f)}$ are unbounded.

Proof. In this case, $F(f)$ is a completely invariant immediate attracting basin and $J(f)$ consists of disjoint simple curves, each with one finite endpoint and the other endpoint at $\infty$; see [13]. The set $I(f)$ consists of the open curves (without endpoints), together with some of their finite endpoints; see [22] and [25]. These open curves are in $Z(f)$, and even in $A(f)$; see [35] and [26]. Thus $M(f)$ is contained in the set of finite endpoints which is totally disconnected (see 24]), so the components of $M(f)$ are singletons. However, the components of $\overline{L(f)}, \overline{M(f)}$ and $\overline{I^{a}(f)}$ are all unbounded, by Theorem 4 (a)(ii).

Example 3. Let

$$
f(z)=z+1+e^{-z} .
$$

Then $f$ has a completely invariant Baker domain $U$ such that $U \subset L(f)$. The sets $L(f), M(f)$ and $I(f)$ are all connected and dense in $\mathbb{C}$, as is $I^{a}(f)$ whenever $\liminf _{n \rightarrow \infty} a_{n} / n>0$. However, all components of $M(f) \cap J(f)$ are singletons, and hence so are those of $L(f) \cap J(f)$ and also $I^{a}(f) \cap J(f)$ when $I^{a}(f) \subset M(f)$.

Proof. In this case, $F(f)$ is a completely invariant Baker domain $U$ in which $f^{n}(z) \rightarrow \infty$ as $n \rightarrow \infty$ and $\left|f^{n}(z)\right|=O(n)$ as $n \rightarrow \infty$; see [17. Example 1]. Thus we have $U \subset L(f) \subset M(f)$ and $U \subset I^{a}(f)$ whenever $\liminf _{n \rightarrow \infty} a_{n} / n>0$. Note, however, that there are points of $\partial U$ (for example, fixed points of $f$ ) which are not in $I(f)$. Each of the sets $L(f), M(f), I^{a}(f)$ and $I(f)$ is connected and dense in $\mathbb{C}$, since $U \subset I^{a}(f) \subset \bar{U}=\mathbb{C}$, for example.

It can be shown by using a result of Barański [5, Theorem 3], together with the fact that $f$ is the lift of $g(w)=(1 / e) w e^{-w}$ under $w=e^{-z}$, that $J(f)$ consists of disjoint simple curves, each with one finite endpoint and the other endpoint at $\infty$, and $I(f) \cap J(f)$ consists of the open curves together with some of their finite endpoints. Moreover, these open curves are in $A(f)$ (see [26]), so $M(f) \cap J(f)$ is contained in the set of finite endpoints and its components are singletons. 
Example 4. Let

$$
f(z)=z+\sin z+2 \pi .
$$

Then every component of $F(f)$ is a bounded wandering domain whose closure is contained in $L(f)$. The sets $I(f)$ and $L(f)$ are connected and dense in $\mathbb{C}$, and $I(f) \cap J(f)$ and $L(f) \cap J(f)$ are connected and unbounded. Similar properties hold for $I^{a}(f)$ whenever $\liminf _{n \rightarrow \infty} a_{n} / n>0$.

Proof. Since the function $f$ and $h(z)=z+\sin z$ are both lifts under $w=e^{i z}$ of

$$
g(w)=w \exp \left(\frac{1}{2}(w-1 / w)\right), \quad z \in \mathbb{C} \backslash\{0\},
$$

we have $F(g)=\exp (i F(f))=\exp (i F(h))$, by a result of Bergweiler 9]. Now $F(g)$ consists of the basin of attraction of the super-attracting fixed point -1 , which is the only singular value of $g$, so $F(f)=F(h)$ is the lift of this basin.

In particular, the point -1 lifts to the points $(2 n+1) \pi, n \in \mathbb{Z}$, which are superattracting fixed points of $h$. The immediate basin of attraction of $\pi$ contains the interval $(0,2 \pi)$ and is bounded, since $h$ maps the boundary of the open rectangle $\{x+i y: 0<x<2 \pi,-3<y<3\}$ outside this rectangle. See [16] for a discussion of the properties of functions such as $g$ and their lifts.

Thus $F(f)=F(h)$ consists of an infinite necklace of congruent bounded Fatou components, say $U_{n}, n \in \mathbb{Z}$, where $(2 n+1) \pi \in U_{n}$, together with the successive pre-images of these components under $h$. The components $U_{n}$ form a wandering orbit under $f$ with $f\left(U_{n}\right)=U_{n+1}$ for $n \in \mathbb{Z}$, in which $f^{n}(z) \rightarrow \infty$ as $n \rightarrow \infty$ and $\left|f^{n}(z)\right|=O(n)$ as $n \rightarrow \infty$. Hence

$$
\mathbb{R} \subset \bigcup_{n \in \mathbb{Z}} \overline{U_{n}} \subset L(f) \text { and } \bigcup_{n \in \mathbb{Z}} \partial U_{n} \subset L(f) \cap J(f) .
$$

Now, the pre-image under $f$ of the real axis consists of the real axis itself and infinitely many pairs of curves tending to $\infty$ at both ends, with each pair passing through a critical point $(2 n+1) \pi, n \in \mathbb{Z}$, and lying in $\left\{z:\left(2 n+\frac{1}{2}\right) \pi<\Re z<\right.$ $\left.\left(2 n+\frac{3}{2}\right) \pi\right\}$. Thus $F(f)$ consists of infinite necklaces of bounded Fatou components, together forming an infinite tree-like structure. Therefore the set

$$
E_{1}=\bigcup\{\bar{U}: U \text { is a Fatou component of } f\}
$$

is connected and dense in $\mathbb{C}$, and $E_{1} \subset L(f) \subset I(f) \subset \mathbb{C}=\overline{E_{1}}$. Hence $I(f)$ and $L(f)$ are connected and dense in $\mathbb{C}$. The set

$$
E_{2}=\bigcup\{\partial U: U \text { is a Fatou component of } f\}
$$

is also connected and unbounded, and

$$
E_{2} \subset L(f) \cap J(f) \subset I(f) \cap J(f) \subset J(f)=\overline{E_{1}} \backslash F(f)=\overline{E_{2}} .
$$

Hence $I(f) \cap J(f)$ and $L(f) \cap J(f)$ are connected and unbounded.

Example 5. Let

$$
f(z)=z+e^{-z}+2 \pi i
$$

Then every component of $F(f)$ is an unbounded wandering domain $U$ contained in $L(f)$ and also in $I^{a}(f)$ whenever $\liminf _{n \rightarrow \infty} a_{n} / n>0$. However, the boundaries of these wandering domains are not contained in $L(f)$ and indeed meet $A(f)$. 
Proof. The function $h(z)=z+e^{-z}$ has congruent unbounded invariant Baker domains $U_{n}, n \in \mathbb{Z}$, such that $2 n \pi i \in U_{n} \subset\{z:(2 n-1) \pi<\Im(z)<(2 n+1) \pi\}$, and $\left|h^{m}(z)\right|=O(m)$ as $m \rightarrow \infty$, for $z \in U_{0}$; see [14. The Fatou set of $h$ consists of these Baker domains and their successive pre-images under $h$, which are all unbounded. Since $J(f)=J(h)$, by [9], the components $U_{n}$ form a wandering orbit under $f$ with $f\left(U_{n}\right)=U_{n+1}$ for $n \in \mathbb{Z}$, in which $f^{n}(z) \rightarrow \infty$ as $n \rightarrow \infty$ and $\left|f^{n}(z)\right|=O(n)$ as $n \rightarrow \infty$. Hence $F(f)=F(h) \subset L(f)$.

It was shown in [3, Theorem 6.1] that $\Gamma_{0}=\{z: \Im z=\pi\} \subset \partial U_{0}$, so $\Gamma_{n}=\{z$ : $\Im z=n \pi\} \subset \partial U_{n}$ for $n \in \mathbb{Z}$. It is easy to check directly that each $\Gamma_{n} \subset Z(f)$, and even that $\Gamma_{n} \subset A(f)$. The result now follows.

Example 6. Let

$$
f(z)=\frac{1}{2}\left(\cos z^{1 / 4}+\cosh z^{1 / 4}\right)=1+\frac{z}{4 !}+\frac{z^{2}}{8 !}+\cdots .
$$

Then $A(f)$ and $I(f)$ are connected, all components of $M(f)$ are bounded, and all components of $\overline{L(f)}, \overline{M(f)}$ and $\overline{I^{a}(f)}$ are unbounded.

Proof. The connectedness of $A(f)$ and $I(f)$ is proved in [32, Corollary 5]. This proof depends on the fact that there is a sequence of continua in $A(f)$ which surround 0 and tend to $\infty$, and this property forces all components of $M(f)$ to be bounded. Also, $f$ has no nested wandering domains; see [32, Section 6]. Hence all the components of $\overline{L(f)}, \overline{M(f)}$ and $\overline{I^{a}(f)}$ are unbounded by Theorem 4 (a)(ii).

Remark. Using results in [32, see Theorem 2, its proof, and Section 6], we can show that there are many transcendental entire functions that have the properties of Example 6, as follows:

- if $f$ is a transcendental entire function and there is a hole in $A(f)$, that is, a bounded domain $G$ such that $\partial G \subset A(f)$ but $G \cap J(f) \neq \emptyset$, then there is a sequence of continua in $A(f)$ which surround 0 and tend to $\infty$, from which it follows that $A(f)$ and $I(f)$ are connected and all the components of $M(f)$ are bounded;

- there are many examples of transcendental entire functions for which there is a hole in $A(f)$ and no nested wandering domains - all such functions must have the properties of Example 6

Note that in 32 the set $A(f)$ is called $B(f)$ because an alternative definition is used.

Example 7. Let

$$
f(z)=2 z+2-\log 2-e^{z} .
$$

Then $f$ has an invariant Baker domain $U$ such that $\bar{U} \backslash\left\{z_{0}\right\} \subset L(f)$, where $z_{0} \in \partial U$ is a fixed point of $f$, and a bounded wandering domain $V$ such that $\bar{V} \subset L(f)$.

Proof. It is shown in [8] that the function $f$ has

- an invariant Baker domain $U$ contained in $\{z: \Re z<0\}$ such that the map $f: U \rightarrow U$ is univalent and $\partial U$ is a Jordan curve through $\infty$;

- a bounded Fatou component $V_{0}$ containing the super-attracting fixed point $\log 2$ 
- bounded Fatou components of the form $V_{k}=\left\{z+2 \pi k i: z \in V_{0}\right\}, k \in \mathbb{Z}$, such that $f\left(V_{k}\right)=V_{2 k}$, for $k \in \mathbb{N}$.

Thus $V=V_{1}$ is a bounded wandering domain such that $\bar{V} \subset L(f)$.

Also, it is easy to check that $\partial U$ meets the real axis at a repelling fixed point $z_{0}$ of $f$ and that

$$
(3 / 2)^{n}|z| \leq\left|f^{n}(z)\right| \leq 3^{n}|z|, \quad \text { for } z \in \bar{U} \cap\{z:|z| \geq 2(3+\log 2)\},
$$

so

$$
\bar{U} \cap\{z:|z| \geq 2(3+\log 2)\} \subset L(f) .
$$

Since $f$ is univalent on $U$, it is conjugate, via a Riemann map, to a Möbius transformation of the unit disc onto itself. Since $\partial U$ is a Jordan curve the Riemann map extends to a homeomorphism on the closed unit disc, so the conjugate Möbius transformation fixes two boundary points, one repelling and one attracting; the latter attracts all points of $\mathbb{C}$ except the repelling fixed point. It follows that $\bar{U} \backslash\left\{z_{0}\right\} \subset I(f)$. Hence, by (8.1), the whole of $\bar{U} \backslash\left\{z_{0}\right\}$ is contained in $L(f)$.

Note that in this case $J(f)$ is connected; see [23].

Our final example shows that Theorem 4(a)(ii) is false without the assumption that $f$ is entire.

\section{Example 8. Let}

$$
f(z)=\lambda \sin z-\varepsilon /(z-\pi), \quad \text { where } 0<\lambda<1, \varepsilon>0 \text { small. }
$$

Then $f$ has a direct tract and no nested wandering domains, and $\overline{L(f)}, \overline{M(f)}$ and $\overline{I^{a}(f)}$ each have infinitely many bounded components.

Proof. The function $f$ has a direct tract, since it has only one pole, and it has a completely invariant, unbounded, infinitely connected, attracting Fatou component; see [14, Example 2]. Hence $J(f)$ has infinitely many bounded components and $f$ has no nested wandering domains. Since $f$ has a direct tract, this is equivalent to saying that $f$ has no quasi-nested wandering domains. Thus the sets $\overline{L(f)}, \overline{M(f)}$ and $\overline{I^{a}(f)}$ have infinitely many bounded components, by Theorem 3 (c) and (d).

\section{ACKNOWLEDGEMENT}

We are grateful to Dr. J. Sivaloganathan, whose question during a colloquium at the University of Bath prompted this work. We would also like to thank the referee for helpful suggestions which led to improvements in the paper.

\section{REFERENCES}

[1] I.N. Baker, An entire function which has wandering domains, J. Austral. Math. Soc. Ser. A, 22 (1976), 173-176. MR0419759 (54:7777)

[2] I.N. Baker, Wandering domains in the iteration of entire functions, Proc. London Math. Soc. (3), 49 (1984), 563-576. MR759304 (86d:58066)

[3] I.N. Baker and P. Domínguez, Boundaries of unbounded Fatou components of entire functions, Ann. Acad. Sci. Fenn. Math., 24 (1999), 437-464. MR1724391(2000j:37055)

[4] I.N. Baker and L.S.O. Liverpool, Picard sets for entire functions, Math. Z., 126 (1972), 230-238. MR0344473(49:9212)

[5] K. Barański, Trees and hairs for entire maps of finite order, Math. Z., 257 (2007), no. 1, 33-59. MR:2318569 (2008f:37097)

[6] A.F. Beardon, Iteration of rational functions, Graduate Texts in Mathematics 132, SpringerVerlag, 1991. MR1128089 (92j:30026) 
[7] W. Bergweiler, Iteration of meromorphic functions, Bull. Amer. Math. Soc., 29 (1993), 151188. MR.1216719 (94c:30033)

[8] W. Bergweiler, Invariant domains and singularities, Math. Proc. Camb. Phil. Soc., 117 (1995), 525-532. MR.1317494 (96b:30055)

[9] W. Bergweiler, On the Julia set of analytic self-maps of the punctured plane, Analysis, 15 (1995), 251-256. MR1357963 (96k:30028)

[10] W. Bergweiler, An entire function with simply and multiply connected wandering domains, Pure Appl. Math. Quarterly, 7 (2011), 107-120.

[11] W. Bergweiler and A. Hinkkanen, On semiconjugation of entire functions, Math. Proc. Camb. Phil. Soc., 126 (1999), 565-574. MR.1684251(2000c:37057)

[12] W. Bergweiler, P.J. Rippon and G.M. Stallard, Dynamics of meromorphic functions with direct or logarithmic singularities, Proc. London Math. Soc., 97 (2008), 368-400. MR 2439666

[13] R.L. Devaney and F. Tangerman, Dynamics of entire functions near the essential singularity, Ergodic Theory Dynam. Systems, 6 (1986), 498-503. MR.873428 (88e:58057)

[14] P. Domínguez, Dynamics of transcendental meromorphic functions, Ann. Acad. Sci. Fenn. Math. Ser. A (1), 23 (1998), 225-250. MR1601879 (99b:30031)

[15] A.E. Eremenko, On the iteration of entire functions, Dynamical systems and ergodic theory, Banach Center Publications 23, Polish Scientific Publishers, Warsaw, 1989, 339-345. MR1102727 (92c:30027)

[16] N. Fagella, Dynamics of the complex standard family, J. Math. Anal. Appl., 229 (1999), 1-31. MR.1664296 (99j:58168)

[17] P. Fatou, Sur l'itération des fonctions transcendantes entières, Acta Math., 47 (1926), 337360. MR 1555220

[18] D. Gaier, Lectures on complex approximation, Birkhäuser, 1985. MR894920 (88i:30059b)

[19] W.K. Hayman, Meromorphic functions, Clarendon Press, Oxford, 1964. MR0164038 $(29: 1337)$

[20] W.K. Hayman and P.B. Kennedy, Subharmonic functions, Volume I, Academic Press, 1976. MR0460672 (57:665)

[21] A. Hinkkanen, Julia sets of polynomials are uniformly perfect, Bull. London Math. Soc., 26 (1994), 153-159. MR1272301 (95c:30031)

[22] B. Karpińska, Hausdorff dimension of the hairs without endpoints for $\lambda \exp (z), C$. R. Acad. Sci. Paris Sér. I Math., 328 (1999), 1039-1044. MR.1696203 (2000e:37054)

[23] M. Kisaka, On the connectivity of Julia sets of transcendental entire functions, Ergodic Theory Dynam. Systems, 18 (1998), 189-205. MR1609471 (99a:30033)

[24] J.C. Mayer, An explosion point for the set of endpoints of the Julia set of $\lambda \exp (z)$, Ergodic Theory Dynam. Systems, 10 (1990), 177-183. MR1053806 (91e:58153)

[25] L. Rempe, Topological dynamics of exponential maps on their escaping sets, Ergodic Theory Dynam. Systems, 26 (2006), 1939-1975. MR2279273 (2008c:37069)

[26] L. Rempe, P.J. Rippon and G.M. Stallard, Are Devaney hairs fast escaping? J. Difference Eq. Appl., 16 (2010), no. 5-6, 739-762. MR2675603

[27] P.J. Rippon, Asymptotic values of continuous functions in Euclidean space, Math. Proc. Camb. Phil. Soc., 111 (1992), 309-318. MR1142750 (92k:30051)

[28] P.J. Rippon, Baker domains of meromorphic functions, Ergodic Theory Dynam. Systems, 26 (2006), 1225-1233. MR2247639 (2008d:37074)

[29] P.J. Rippon and G.M. Stallard, On sets where iterates of a meromorphic function zip towards infinity, Bull. London Math. Soc., 32 (2000), 528-536. MR1767705 (2001g:30019)

[30] P.J. Rippon and G.M. Stallard, On questions of Fatou and Eremenko, Proc. Amer. Math. Soc., 133 (2005), 1119-1126. MR2117213(2005j:37069)

[31] P.J. Rippon and G.M. Stallard, On multiply connected wandering domains of meromorphic functions, J. London Math. Soc., 77 (2008), 405-423. MR2400399 (2009a:37093)

[32] P.J. Rippon and G.M. Stallard, Escaping points of entire functions of small growth, Math. Z., 261 (2009), 557-570. MR2471088

[33] P.J. Rippon and G.M. Stallard, Functions of small growth with no unbounded Fatou components, J. Anal. Math., 108 (2009), 61-86. MR2544754

[34] P.J. Rippon and G.M. Stallard, Fast escaping points of entire functions, arXiv:1009.5081.

[35] D. Schleicher and J. Zimmer, Escaping points of exponential maps, J. London Math. Soc. (2), 67 (2003), 380-400. MR.1956142 (2003k:37067) 
[36] M. Tsuji, Potential theory in modern function theory, Maruzen, Tokyo, 1959; reprint by Chelsea, New York, 1975. MR0114894 (22:5712)

[37] J-H. Zheng, On uniformly perfect boundary of stable domains in iteration of meromorphic functions II, Math. Proc. Camb. Phil. Soc., 132 (2002), 531-544. MR.1891688(2003b:37069)

[38] J-H. Zheng, On multiply-connected Fatou components in iteration of meromorphic functions, J. Math. Anal. Appl., 313 (2006), 24-37. MR2178719(2006e:37076)

Department of Mathematics and Statistics, The Open University, Walton Hall, MilTON Keynes MK7 6AA, United Kingdom

E-mail address: p.j.rippon@open.ac.uk

Department of Mathematics and Statistics, The Open University, Walton Hall, MilTON Keynes MK7 6AA, United Kingdom

E-mail address: g.m.stallard@open.ac.uk 